Алгебра и анализ

Том. 16 (2004), вып. 5
St. Petersburg Math. J.

Vol. 16 (2005), No. 5, Pages 791-813

S 1061-0022(05)00879-4

Article electronically published on September 21, 2005

\title{
TWIST NUMBER OF (CLOSED) BRAIDS
}

\author{
A. V. MALYUTIN
}

\begin{abstract}
A real-valued invariant of (closed) braids, called the twist number, is introduced and studied. This invariant is effectively computable and has clear geometric sense.

As a functional on the braid group, the twist number is a pseudocharacter (i.e., a function that is "almost" a homomorphism). It is closely related to Dehornoy's ordering (and to all Thurston-type orderings) on the braid group. In special cases, the twist number coincides with some characteristics introduced by William Menasco. In terms of the twist number, restrictions are established on the applicability of the Markov destabilization and Birman-Menasco moves on closed braids. These restrictions were conjectured by Menasco (Kirby's problem book, 1997). As a consequence, conditions for primality of the link represented by a braid are obtained.

The results were partially announced in an earlier paper.
\end{abstract}

\section{INTRODUCTION}

In this paper we introduce a new real-valued invariant on the braid group $B_{n}$. We call this invariant the twist number and denote it by $\omega$ :

$$
\omega: B_{n} \rightarrow \mathbb{R} \text {. }
$$

The choice of the term "twist number" is motivated by the fact that, in a sense, the invariant $\omega$ characterizes how much a braid is "twisted" or "wound" from its exterior. For example, the twist number of any split braid is equal to 0. (Indeed, in a sense, it is natural to regard a split braid as "nontwisted".) The twist number of the full twist equals $1\left(\omega\left(\Delta^{2}\right)=1\right)$; furthermore, for each $\beta \in B_{n}$ we have $\omega\left(\beta \Delta^{2}\right)=\omega(\beta)+1$.

The function $\omega: B_{n} \rightarrow \mathbb{R}$ is a pseudocharacter (see the definitions in $\S 6$ ). In particular, for any $\beta_{1}, \beta_{2} \in B_{n}$ we have the inequality

$$
\left|\omega\left(\beta_{1} \beta_{2}\right)-\omega\left(\beta_{1}\right)-\omega\left(\beta_{2}\right)\right| \leq 1
$$

The restriction of the twist number to any Abelian subgroup of $B_{n}$ is a homomorphism. For instance, for any $\beta \in B_{n}$ and any $k \in \mathbb{Z}$ we have $\omega\left(\beta^{k}\right)=k \omega(\beta)$.

It is well known that there is a one-to-one correspondence between the conjugacy classes in braid groups and the isotopy classes of closed braids. Like any other pseudocharacter, the twist number is a conjugacy invariant. Hence, the twist number of a closed braid is well defined: $\omega(\widehat{\beta}):=\omega(\beta)$.

The twist number is closely related to Thurston-type orderings on the braid group, including Dehornoy's vastly known ordering (see the definitions in [5]). In $\S 7$, we establish some relations between the twist number and Dehornoy's ordering. (The same relations are valid for the twist number and any other Thurston-type ordering.) In particular, we deduce a formula expressing the twist number in terms of the ordering (see Theorem

2000 Mathematics Subject Classification. Primary 57M25, 57M50.

Key words and phrases. Braid group, Markov destabilization, Birman-Menasco moves, link theory.

This work was partly supported by grant PD02-1.1-423 from Russian Ministry of Education and by grant NSh-1914.2003.1 from President of Russia (Support of Leading Scientific Schools). 
7.5). We also describe a method of computation of the twist number for a given braid, which involves algorithms of comparisons of braids in Dehornoy's ordering.

The twist number is a real-valued invariant. Moreover, we show that it is rationalvalued (Theorem 11.1). The proof of this result (see §11) involves the Nielsen-Thurston classification of surface automorphisms.

The twist number can be defined in terms of the Nielsen-Thurston theory. Using this theory, Menasco [10] defined some characteristics for braids of periodic and pseudoAnosov types. In terms of those characteristics, Menasco formulated four conjectures concerning the admissibility of the Markov destabilization, and also of exchange move and flype (two braid moves introduced by Birman and Menasco) for a closed braid. It turns out that, for both types of braids (periodic and pseudo-Anosov), Menasco's characteristics are equal to the twist number (see the definitions in [10] and in $\S 11$ below). Theorem 14.2 below confirms all four conjectures, and some of them in a stronger form (with the exception of the conjecture on periodic braids in the part concerning the flype: this part was disproved in [11]).

In a natural way, the notion of the twist number (as well as some other methods and results of the present paper) can be generalized to the case of the mapping class group of any surface with nonempty boundary (we recall that the braid group is isomorphic to the mapping class group of a punctured disk). The case of a punctured disk (i.e., the "usual" braid group) is of most interest for us because the twist number on the braid group is applicable in the theory of knots in $\mathbb{R}^{3}$.

By the Alexander theorem, each link type in $\mathbb{R}^{3}$ can be represented by a closed braid. Closed braids representing the same link may have different twist numbers, so that the twist number is not an invariant of a link. Nevertheless, the twist number of a braid provides some information on the corresponding link. For example, the link represented by a closed braid $\widehat{\beta}$ is prime whenever $|\omega(\widehat{\beta})|>1$ (Theorem 15.3).

Structure of the paper. $\S 1$ contains the definitions of the braid group $B_{n}$ and of the mapping class group of a punctured disk.

$\S 2$ is devoted to the study of the natural action of the braid group on the real line; we call this the Nielsen-Thurston action.

In $\S 3$, we define the twist number in terms of the Nielsen-Thurston action and the translation number. It should be noted that the twist number can be defined in several essentially different ways. The definition in $\S 3$ is somewhat bulky, but we use it as a basic one for the convenience of proofs. The reader can formulate more compact definitions of the twist number on the basis of Theorem 7.5, or of the uniqueness theorem (Theorem 8.1).

In $\S 4$, we deduce auxiliary "formulas" describing the twist number in terms of the action of the braid group on the free group, which is known as the Artin action.

In $\S 5$, we prove the basic properties of the twist number.

In $\S 6$, we recall the definition of a pseudocharacter and show that the twist number on the braid group is a pseudocharacter. We also formulate the corresponding properties of the twist number and find its defect.

In $\S 7$, the definition of Dehornoy's ordering is presented, and some relations between this ordering and the twist number are proved. Theorem 7.5 expresses the twist number in terms of this ordering.

In $\S 8$, we prove the uniqueness theorem, which contains a list of properties of the twist number that determines it uniquely.

In $\S 9$, we recall the definition of a closed braid and introduce the notion of the twist number of a closed braid. 
In $\S 10$, we introduce the notion of a satellite braid and show that the twist number of a braid coincides with that of any of its satellites.

In $\S 11$, we use the Nielsen-Thurston classification of the automorphisms of surfaces in order to prove that the twist number of any braid is a rational number (Theorem 11.1).

In $\S 12$, we describe a method for computation of the twist number.

In $\S 13$, we estimate the twist number of a braid by the number of occurrences of a generator in the corresponding braid word. One of these estimates is used in the proof of Theorem 14.2.

$\S 14$ is devoted to transformations of closed braids. Here, we present the definitions of destabilization, exchange move, and flype. In Theorem 14.2, in terms of the twist number, we establish restrictions on the possibility of performing the above transformations.

In $\S 15$, sufficient conditions of primality of a link represented by a braid are given in terms of the twist number (Theorem 15.3).

\section{$\S 1$. BRAID GROUP}

1.1. Braid group. The Artin braid group $B_{n}$ on $n$ strands is defined by the presentation

$$
B_{n}:=\left\langle\sigma_{1}, \ldots, \sigma_{n-1}\left|\sigma_{i} \sigma_{j}=\sigma_{j} \sigma_{i},\right| i-j \mid \geq 2 ; \sigma_{i} \sigma_{i+1} \sigma_{i}=\sigma_{i+1} \sigma_{i} \sigma_{i+1}\right\rangle .
$$

The group $B_{1}$ is trivial, while $B_{2} \cong \mathbb{Z}$. The elements of $B_{n}$ are called braids. The generators $\sigma_{1}^{ \pm 1}, \ldots, \sigma_{n-1}^{ \pm 1}$ are Artin's generators. Further on, by a braid word we mean a word in Artin's generators.

The braid

$$
\Delta:=\left(\sigma_{1} \sigma_{2} \cdots \sigma_{n-1}\right)\left(\sigma_{1} \sigma_{2} \cdots \sigma_{n-2}\right) \cdots\left(\sigma_{1} \sigma_{2}\right)\left(\sigma_{1}\right) \in B_{n}
$$

is called the fundamental braid. The braid $\Delta^{2} \in B_{n}$ generates the center of the group $B_{n}$ whenever $n>2$. (The center of $B_{n}$ is an infinite cyclic group.)

1.2. The mapping class group of the punctured disk. We denote by $D^{2}$ the closed unit disk with center 0 in the complex plane $\mathbb{C}$, and we denote by $D_{n}$ the disk $D^{2}$ from which $n$ distinct points on the real interval $(-1 ; 1)$ are removed.

The mapping class group of $D_{n}$ is the group

$$
\operatorname{MCG}\left(D_{n}\right):=\operatorname{Homeo}_{+}\left(D_{n}, \partial D_{n}\right) / \operatorname{Homeo}_{0}\left(D_{n}, \partial D_{n}\right),
$$

where Homeo $_{+}\left(D_{n}, \partial D_{n}\right)$ is the group consisting of all orientation-preserving homeomorphisms of $D_{n}$ that fix $\partial D_{n}$ pointwise, and $\operatorname{Homeo}_{0}\left(D_{n}, \partial D_{n}\right)$ is the normal subgroup consisting of all homeomorphisms isotopic to the identity rel $\partial D_{n}$.

1.3. Isomorphism $B_{n} \cong \operatorname{MCG}\left(D_{n}\right)$. It is well known that $B_{n}$ is isomorphic to $\operatorname{MCG}\left(D_{n}\right)$ (see, e.g., [1]). The canonical isomorphism $B_{n} \cong \operatorname{MCG}\left(D_{n}\right)$ can be constructed as follows: we enumerate the punctures by $1, \ldots, n$ from left to right. After that, the generator $\sigma_{i} \in B_{n}$ is associated with the class in $\operatorname{MCG}\left(D_{n}\right)$ of the autohomeomorphism that exchanges the punctures $i$ and $i+1$ by "rotating" them clockwise in the simplest possible way (this is a Dehn half-twist).

\section{§2. Nielsen-Thurston Representation}

In this section, we describe a natural action of the group $B_{n} \cong \operatorname{MCG}\left(D_{n}\right)$ on the real line by order-preserving autohomeomorphisms, i.e., a homomorphism

$$
\Omega: B_{n} \cong \operatorname{MCG}\left(D_{n}\right) \rightarrow \operatorname{Homeo}_{+}(\mathbb{R}) .
$$

We call this homomorphism the Nielsen-Thurston representation or the NielsenThurston action. We use this action to define the twist number of a braid (see §3). 
The description of the Nielsen-Thurston action in the general case of a mapping class group of any surface with nonempty boundary can be found in [14, 17.

2.1. Compactification of the universal covering space and the hyperbolic structure. To describe the Nielsen-Thurston action, we need the following construction. Let $D_{n}$ denote the disk with $n$ punctures, and let $\mathcal{U}$ be a universal covering space of $D_{n}$. It is well known that $D_{n}$ can be equipped with a hyperbolic metric (provided $n \geq 2$ ) in which the circle $\partial D_{n}$ is a geodesic and the punctures are cusps. This metric on $D_{n}$ lifts to a hyperbolic metric on $\mathcal{U}$. Being simply connected, $\mathcal{U}$ can be isometrically embedded in the hyperbolic plane $\mathbb{H}^{2}$, and we identify $\mathcal{U}$ with a subset of $\mathbb{H}^{2}$. The boundary of $\mathcal{U} \subset \mathbb{H}^{2}$ (it is the inverse image of $\partial D_{n}$ ) is a union of disjoint geodesics in $\mathbb{H}^{2}$, and $\mathbb{H}^{2} \backslash \mathcal{U}$ is a union of disjoint open half-planes. (In particular, it follows that $\mathcal{U}$ is a convex subset in $\mathbb{H}^{2}$.) We denote by $\overline{\mathbb{H}}^{2}$ the standard compactification of the hyperbolic plane, i.e., the closed disk obtained from $\mathbb{H}^{2}$ by adding a circle at infinity (absolute). Let $\mathcal{D}$ be the closure of $\mathcal{U}$ in $\overline{\mathbb{H}}^{2}$, i.e., a compactification of the space $\mathcal{U}$. Since $\mathcal{U}$ is convex, $\mathcal{D}$ is homeomorphic to a closed disk. Here, we have $\mathcal{D} \cap \mathbb{H}^{2}=\mathcal{U}$, and the set $\mathcal{K}:=\mathcal{D} \backslash \mathcal{U}=\mathcal{D} \cap \partial \overline{\mathbb{H}}^{2}$ of the limit points of $\mathcal{U}$ is a Cantor set on the absolute.

2.2. Remark. We observe that the resulting compactification $\mathcal{D}$ has the following property: the boundary $\partial \mathcal{U}$ of the universal covering is dense in $\partial \mathcal{D}$. It can be proved that this compactification is "canonical" (in particular, it does not depend on the choice of a hyperbolic structure) in the following sense. Suppose $D^{\prime}$ is a closed disk and $f: \mathcal{U} \rightarrow D^{\prime}$ is an embedding such that $f(\operatorname{int}(\mathcal{U}))=\operatorname{int}\left(D^{\prime}\right)$ and the set $f(\partial \mathcal{U})$ is dense in $\partial D^{\prime}$. Then $f$ is uniquely extended to a homeomorphism between $\mathcal{D}$ and $D^{\prime}$. This property implies that each autohomeomorphism of $\mathcal{U}$ is uniquely extended to an autohomeomorphism of the entire disk $\mathcal{D}$. In particular, each lifting of any autohomeomorphism of the punctured disk $D_{n}$ is uniquely extended to an autohomeomorphism of $\mathcal{D}$. The latter fact, which is involved in the construction of the Nielsen-Thurston representation, is proved in a standard way with the help of the methods of hyperbolic geometry (see, e.g., [3]).

2.3. Notation. Suppose $A$ is a topological space, $f: A \rightarrow A$ is an autohomeomorphism of $A$, and $B \subset A$ is a subspace such that $f(B)=B$. We denote by $\left.f\right|_{B}$ the autohomeomorphism of $B$ obtained by restricting $f$ :

$$
\left.f\right|_{B}: B \rightarrow B,\left.\quad f\right|_{B}(x)=f(x) .
$$

2.4. The Nielsen-Thurston action. Now we pass to a description of the NielsenThurston action.

We let $\mathcal{I} \subset \partial \mathcal{U}$ be one of the geodesics in $\partial \mathcal{U}$, i.e., a connected component of the preimage of the circle $\partial D_{n}$. Any autohomeomorphism $\phi: D_{n} \rightarrow D_{n}$ identical on the boundary has a unique lifting $\phi^{\sim}: \mathcal{U} \rightarrow \mathcal{U}$ identical on $\mathcal{I}$, and we obtain a homomorphism

$$
\mathrm{Homeo}_{+}\left(D_{n}, \partial D_{n}\right) \rightarrow \mathrm{Homeo}_{+}(\mathcal{U}), \quad \phi \mapsto \phi^{\sim} .
$$

The autohomeomorphism $\phi^{\sim}$ is uniquely extended to an autohomeomorphism $\phi^{\bar{\sim}}$ of the disk $\mathcal{D}$. (The proof is standard; see, e.g., [3.)

Next, we observe that the autohomeomorphism $\left.\phi^{\sim}\right|_{\partial \mathcal{D}}$ is completely determined by the class $[\phi] \in \operatorname{MCG}\left(D_{n}\right)$. Indeed, we easily check that for each autohomeomorphism $\psi \in \operatorname{Homeo}_{0}\left(D_{n}, \partial D_{n}\right)$ the autohomeomorphism $\left.\psi^{\sim}\right|_{\partial \mathcal{U}}$ is an identity, and since the set $\partial \mathcal{U}$ is dense in $\partial \mathcal{D}$, the autohomeomorphism $\left.\psi^{\bar{\sim}}\right|_{\partial \mathcal{D}}$ is also an identity.

Thus, we have defined a homomorphism

$$
\Omega_{\circ}: \operatorname{MCG}\left(D_{n}\right) \rightarrow \mathrm{Homeo}_{+}(\partial \mathcal{D}),\left.\quad[\phi] \mapsto \phi^{\bar{\sim}}\right|_{\partial \mathcal{D}} .
$$


The geodesic $\mathcal{I}$ is an arc of the circle $\partial \mathcal{D}$. We denote by $\mathcal{J}$ the set $\partial \mathcal{D} \backslash \overline{\mathcal{I}}$, which is homeomorphic to an open interval. Since the action $\Omega_{\circ}$ is identical on $\mathcal{I}$ and on the closure $\overline{\mathcal{I}}$, we arrive at an action of the group $\operatorname{MCG}\left(D_{n}\right)$ on $\mathcal{J}$ : it is the homomorphism

$$
\Omega: \operatorname{MCG}\left(D_{n}\right) \rightarrow \operatorname{Homeo}_{+}(\mathcal{J}),\left.\quad[\phi] \mapsto \phi^{\bar{\sim}}\right|_{\mathcal{J}} .
$$

It can be shown that the action obtained is faithful (i.e., $\Omega$ is a monomorphism).

2.5. Remark. When considering the action $\Omega$, it is convenient to fix a hyperbolic metric on $D_{n}$; however, actually, $\Omega$ does not depend on the choice of the metric. Moreover, this action can be described in nonhyperbolic terms, for example, in terms of ordered sets: we observe that the subset $\partial \mathcal{U}$ of the circle $\partial \mathcal{D}$ is cyclically ordered in a natural way, while the set $\partial \mathcal{U} \backslash \mathcal{I}$ is linearly ordered. It is not hard to prove that these two orders (the cyclic order on $\partial \mathcal{U}$ and the linear order on $\partial \mathcal{U} \backslash \mathcal{I}$ ) do not depend on the hyperbolic structure. The interval $\mathcal{J}$ can be defined as the Dedekind completion of the linearly ordered set $\partial \mathcal{U} \backslash \mathcal{I}$. For each autohomeomorphism $\phi \in \operatorname{Homeo}_{+}\left(D_{n}, \partial D_{n}\right)$, the autohomeomorphism $\left.\phi^{\sim}\right|_{\partial \mathcal{U} \backslash \mathcal{I}}$ (which, obviously, is completely determined by the class $[\phi] \in \operatorname{MCG}\left(D_{n}\right)$ ) preserves the linear order on $\partial \mathcal{U} \backslash \mathcal{I}$ and, therefore, is uniquely extended to an order automorphism of the Dedekind completion $\mathcal{J}$. Such extensions to the Dedekind completion yield the homomorphism $\Omega: \operatorname{MCG}\left(D_{n}\right) \rightarrow \mathrm{Homeo}_{+}(\mathcal{J})$.

\section{§3. Definition of the twist number}

The definition of the twist number given below is based upon the notions of the Nielsen-Thurston action and the translation number. We use the construction and definitions of the preceding section.

3.1. The basic definition is outlined like this: using the Nielsen-Thurston representation, we obtain a representation

$$
\widetilde{\Omega}: B_{n} \cong \operatorname{MCG}\left(D_{n}\right) \rightarrow \widetilde{\mathrm{Homeo}_{+}}\left(\mathbb{S}^{1}\right),
$$

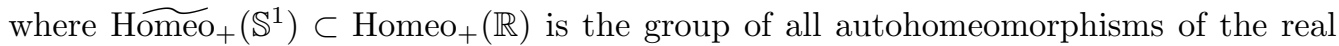
line that commute with the integral translations (see Subsection 3.2).

Then we define the twist number on the group $B_{n} \cong \operatorname{MCG}\left(D_{n}\right)$ as the composition

$$
\omega:=\tau \circ \widetilde{\Omega}: B_{n} \cong \operatorname{MCG}\left(D_{n}\right) \rightarrow \mathbb{R},
$$

where $\tau: \widetilde{H}^{\text {omeo }}+\left(\mathbb{S}^{1}\right) \rightarrow \mathbb{R}$ is the translation number (see Subsection 3.5).

The following commutative diagram, where the rows are exact sequences, clarifies our construction. (The definitions of the objects involved are given in Subsections 3.2 and 3.3.)

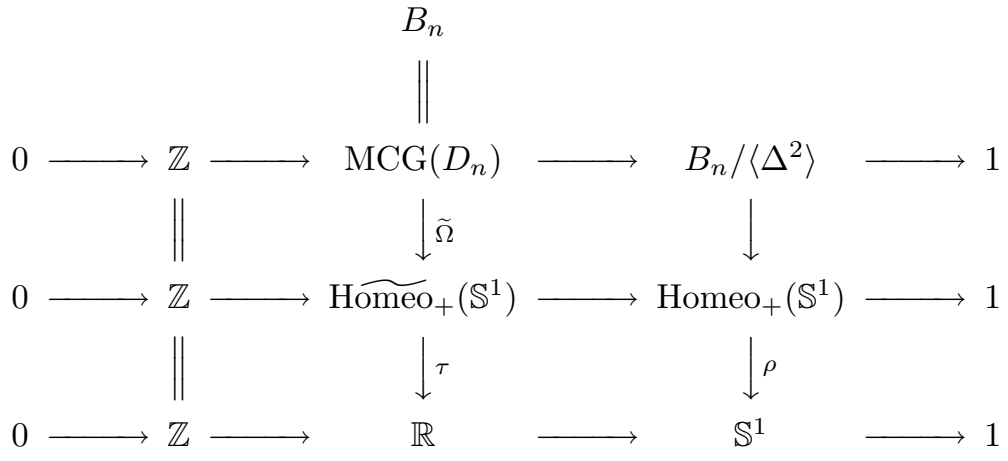


3.2. The group Homeo $+\left(\mathbb{S}^{1}\right)$. We denote by Homeo $+\left(\mathbb{S}^{1}\right)$ the group of all autohomeomorphisms of the real line that commute with the translation $t_{1}$ through 1: $a \mapsto a+1$, i.e., $g \in \mathrm{Homeo}_{+}\left(\mathbb{S}^{1}\right)$ if and only if $g(a+1)=g(a)+1$ for all $a \in \mathbb{R}$.

3.3. Homomorphism $\widetilde{\Omega}: \operatorname{MCG}\left(D_{n}\right) \rightarrow \mathrm{Homeo}_{+}\left(\mathbb{S}^{1}\right)$. Observe that each homeomorphism $i: \mathcal{J} \rightarrow \mathbb{R}$ of the interval $\mathcal{J}$ onto the real line induces an isomorphism

$$
i_{*}: \mathrm{Homeo}_{+}(\mathcal{J}) \rightarrow \mathrm{Homeo}_{+}(\mathbb{R}), \quad t \mapsto i \circ t \circ i^{-1},
$$

and the corresponding action

$$
\Omega_{i}:=i_{*} \circ \Omega: \operatorname{MCG}\left(D_{n}\right) \rightarrow \mathrm{Homeo}_{+}(\mathbb{R})
$$

of the group $\operatorname{MCG}\left(D_{n}\right)$ on $\mathbb{R}$.

It turns out that for a certain choice of $i$ the image of the homomorphism $\Omega_{i}$ lies in the group $\widetilde{\mathrm{HomeO}}_{+}\left(\mathbb{S}^{1}\right)$.

Consider the orientation-preserving isometry of $\mathbb{H}^{2}$ that shifts $\mathcal{I}$ in the positive direction (that is, the direction induced by the clockwise direction on $\partial D_{n}$ ) through a distance equal to the length of the circle $\partial D_{n}$ (in the fixed hyperbolic metric). We denote by $\Theta$ the extension of this isometry to $\overline{\mathbb{H}}^{2}$. Clearly, the restriction $\left.\Theta\right|_{\mathcal{U}}$ is a deck transformation. The homeomorphism $\Theta$ maps $\mathcal{U}$ onto $\mathcal{U}, \mathcal{D}$ onto $\mathcal{D}, \mathcal{I}$ onto $\mathcal{I}$, and, hence, $\mathcal{J}(=\partial \mathcal{D} \backslash \overline{\mathcal{I}})$ onto $\mathcal{J}$. This means that the restriction $\left.\Theta\right|_{\mathcal{J}}: \mathcal{J} \rightarrow \mathcal{J}$ is an autohomeomorphism.

Claim 1. There is a homeomorphism $i: \mathcal{J} \rightarrow \mathbb{R}$ such that $i_{*}\left(\left.\Theta\right|_{\mathcal{J}}\right)=t_{1}$.

Proof. Indeed, since $\left.\Theta\right|_{\mathcal{U}}$ is an isometry of hyperbolic type, $\left.\Theta\right|_{\mathcal{U}}$ has exactly two fixed points in $\overline{\mathbb{H}}^{2}$, which lie in the set $\overline{\mathcal{I}} \cap \overline{\mathcal{J}}$. It follows that the autohomeomorphism $\left.\Theta\right|_{\mathcal{J}}$ has no fixed points. Consequently, the quotient space $\mathcal{J} /\left.\Theta\right|_{\mathcal{J}}$, which consists of the orbits of the action of $\left.\Theta\right|_{\mathcal{J}}$ on $\mathcal{J}$, is a circle. We regard the interval $\mathcal{J}$ as the universal covering space of the circle, and $\left.\Theta\right|_{\mathcal{J}}$ as a deck transformation. Similarly, the translation $t_{1}$ through 1 has no fixed points in $\mathbb{R}$, the quotient space $\mathbb{R} / t_{1}$ is the unit circle $\mathbb{S}^{1}$ with universal covering space $\mathbb{R}$, and $t_{1}$ is a deck transformation. We fix an arbitrary homeomorphism between the circles $\mathcal{J} /\left.\Theta\right|_{\mathcal{J}}$ and $\mathbb{R} / t_{1}$ and lift it to a homeomorphism $i: \mathcal{J} \rightarrow \mathbb{R}$. Obviously, the map $i_{*}$ takes the autohomeomorphism $\left.\Theta\right|_{\mathcal{J}}$ either to $t_{1}$ (then $i$ is the required homeomorphism) or to $t_{1}^{-1}$, and then the imposed requirements are fulfilled, for example, for the homeomorphism $s \circ i$, where $s$ is the automorphism $\mathbb{R} \rightarrow \mathbb{R}: a \mapsto-a$.

Now, let $j: \mathcal{J} \rightarrow \mathbb{R}$ be a homeomorphism such that $j_{*}\left(\left.\Theta\right|_{\mathcal{J}}\right)=t_{1}$. Consider the homeomorphism

$$
\widetilde{\Omega}:=\Omega_{j}=j_{*} \circ \Omega: \operatorname{MCG}\left(D_{n}\right) \rightarrow \text { Homeo }_{+}(\mathbb{R}) .
$$

Claim 2. We have $\operatorname{Im} \widetilde{\Omega} \subset \mathrm{Homeo}_{+}\left(\mathbb{S}^{1}\right)$.

Proof. Obviously, the deck transformation $\left.\Theta\right|_{\mathcal{U}}$ commutes with the liftings of the autohomeomorphisms of $D_{n}$ identical on $\mathcal{I}$. Hence, for each $\phi \in \operatorname{Homeo}_{+}\left(D_{n}, \partial D_{n}\right)$, the autohomeomorphism $\phi^{\bar{\sim}}$ commutes with $\left.\Theta\right|_{\mathcal{D}}$. Consequently, for each $g \in \operatorname{MCG}\left(D_{n}\right)$ the autohomeomorphism $\Omega(g): \mathcal{J} \rightarrow \mathcal{J}$ commutes with the autohomeomorphism $\left.\Theta\right|_{\mathcal{J}}$ : $\mathcal{J} \rightarrow \mathcal{J}$. In other notation, an element $\widetilde{\Omega}(g)$ commutes with the translation through $1, t_{1}=j_{*}(\Theta \mid \mathcal{J})$. This precisely means that the image of the homomorphism $\widetilde{\Omega}$ lies in $\mathrm{HomeO}_{+}\left(\mathbb{S}^{1}\right)$.

Thus, we have constructed a homomorphism

$$
\widetilde{\Omega}: \operatorname{MCG}\left(D_{n}\right) \rightarrow \widetilde{\mathrm{Homeo}_{+}}\left(\mathbb{S}^{1}\right) .
$$


3.4. Remark. The choice of the homeomorphism $j: \mathcal{J} \rightarrow \mathbb{R}$ in the above construction allows some flexibility. It can be shown that in the framework of the construction, the homomorphism $\widetilde{\Omega}=\Omega_{j}$ is determined up to conjugation by an element in Homeo $+\left(\mathbb{S}^{1}\right)$. Namely, if $j^{\prime}: \mathcal{J} \rightarrow \mathbb{R}$ is a homeomorphism such that $j_{*}^{\prime}(\Theta \mid \mathcal{J})=t_{1}$, then $j^{\prime} \circ j^{-1} \in \mathrm{Homeo}_{+}\left(\mathbb{S}^{1}\right)$ and $\Omega_{j^{\prime}}(g)=h\left(\Omega_{j}(g)\right) h^{-1}$, where $h=j^{\prime} \circ j^{-1}$. Conversely, if $h \in \mathrm{HomeO}_{+}\left(\mathbb{S}^{1}\right)$, then the homeomorphism $h \circ j: \mathcal{J} \rightarrow \mathbb{R}$ takes the autohomeomorphism $\left.\Theta\right|_{\mathcal{J}}$ to the translation through 1 , whence $\operatorname{Im} \Omega_{h \circ j} \subset \widetilde{\mathrm{HomeO}_{+}}\left(\mathbb{S}^{1}\right)$. Furthermore, for each $g \in \operatorname{MCG}\left(D_{n}\right)$ we have $\Omega_{h \circ j}(g)=h\left(\Omega_{j}(g)\right) h^{-1}$.

3.5. Poincaré invariants: the translation number. It is well known that for any $\tilde{f} \in \mathrm{Homeo}_{+}\left(\mathbb{S}^{1}\right)$ and $a \in \mathbb{R}$ there exists a finite limit

$$
\tau(\tilde{f}):=\lim _{s \rightarrow \infty} \frac{\tilde{f}^{s}(a)-a}{s}=\lim _{s \rightarrow \infty} \frac{\tilde{f}^{s}(a)}{s} .
$$

Furthermore, for a given $\tilde{f}$ this limit does not depend on the choice of $a$ (see, e.g., 7]). The number $\tau(\tilde{f})$ is called the translation number of the autohomeomorphism $\tilde{f}$.

Usually, the translation number is considered together with the rotation number (the latter taking values in $\left.\mathbb{S}^{1}=\mathbb{R} / \mathbb{Z}\right)$. The rotation number of an autohomeomorphism $f \in$ Homeo $_{+}\left(\mathbb{S}^{1}\right)$ is defined as $\rho(f):=\tau(\tilde{f}) \bmod \mathbb{Z}$, where $\tilde{f} \in \mathrm{Homeo}_{+}\left(\mathbb{S}^{1}\right)$ is a lifting of $f$.

The properties of the Poincaré invariants are well known (see, e.g., [7]).

3.6. Definition. We define the twist number $\omega$ on the group $B_{n} \cong \operatorname{MCG}\left(D_{n}\right)$ as the composition

$$
\omega:=\tau \circ \widetilde{\Omega}: B_{n} \rightarrow \mathbb{R},
$$

where $\widetilde{\Omega}$ is the homomorphism described above and $\tau$ is the translation number.

Thus, the twist number $\omega(\beta)$ of a braid $\beta$ with the corresponding mapping class $g \in \operatorname{MCG}\left(D_{n}\right)$ is defined as follows (here $y \in \mathbb{R}$ is arbitrary):

$$
\omega(\beta):=\omega(g):=\tau(\widetilde{\Omega}(g))=\lim _{s \rightarrow \infty} \frac{(\widetilde{\Omega}(g))^{s}(y)}{s} .
$$

3.7. The consistency of the definition. The constructions preceding the definition admit some flexibility in the choice of a hyperbolic metric on $D_{n}$, of the arc $\mathcal{I}$, and of the homeomorphism transforming $\left.\Theta\right|_{\mathcal{J}}$ into a unit shift. However, the object to be defined, i.e., the twist number, does not depend on this arbitrariness. This fact can be proved "directly", by using the assertions formulated in Remarks 2.2, 2.5, and 3.4, but we can avoid doing this here because below we show that the above definition implies some properties of the twist number that determine it uniquely (see Theorem 8.1) and are formulated in braid group terms. (Thus, these properties are invariant under the choice of the ingredients mentioned above.)

3.8. Remark. The constructions presented above for the punctured disk can be implemented without essential changes in the case of any surface $M$ with nonempty boundary. In particular, let $\operatorname{MCG}(M)$ be the group of isotopy classes of automorphisms fixing the boundary pointwise. Acting in a similar way, we can construct a representation $\operatorname{MCG}(M) \rightarrow \mathrm{Homeo}_{+}\left(\mathbb{S}^{1}\right)$ and also define an invariant similar to the twist number.

\section{$\S 4$. The twist number and the Fundamental group}

In this section, we deduce a "formula" expressing the twist number in terms of the action of the group $B_{n} \cong \operatorname{MCG}\left(D_{n}\right)$ on the fundamental group of the punctured disk $D_{n}$ (Proposition 4.3). We use the constructions and notation of $\S \S 1$ and 2. 
4.1. Action of $\operatorname{MCG}\left(D_{n}\right)$ on $\pi_{1}\left(D_{n}, x_{0}\right)$. We consider the natural (left) action of the group $\operatorname{MCG}\left(D_{n}\right)$ on the fundamental group $\pi_{1}\left(D_{n}, x_{0}\right)$, where $x_{0} \in \partial D_{n}$. We denote by $g v$ the result of the action of a class $g \in \operatorname{MCG}\left(D_{n}\right)$ on an element $v \in \pi_{1}\left(D_{n}, x_{0}\right)$.

Let $u$ be the homotopy class of the loop having endpoints at $x_{0}$ and tracing the circle $\partial D_{n}$ once in the positive direction of the fixed orientation (see Subsection 3.3). In what follows, $u$ is called the distinguished element.

It is easily seen that the powers of $u$ exhaust all $\operatorname{MCG}\left(D_{n}\right)$-invariant elements in $\pi_{1}\left(D_{n}, x_{0}\right)$. We set

$$
\check{\pi}:=\check{\pi}_{1}\left(D_{n}, x_{0}\right):=\pi_{1}\left(D_{n}, x_{0}\right) \backslash\left\{\ldots, u^{-1}, u^{0}=e, u^{1}, u^{2}, \ldots\right\} .
$$

4.2. The ordering of the fundamental group. Let $\widetilde{X}$ denote the inverse image of the point $x_{0}$ in the universal covering (note that $\widetilde{X} \subset \partial \mathcal{U}$ ). We choose a base point $\tilde{x}_{0} \in \tilde{X} \cap \mathcal{I}$ of this preimage (we let $\tilde{x}_{0}$ lie on the $\operatorname{arc} \mathcal{I}$; see the notation in $\S 2$ ).

Let

$$
\mathfrak{a}: \pi_{1}\left(D_{n}, x_{0}\right) \rightarrow \widetilde{X}
$$

be the bijection that takes each element $v \in \pi_{1}\left(D_{n}, x_{0}\right)$ to the endpoint of a curve $\gamma:[0,1] \rightarrow \mathcal{U}$, where $\gamma$ is a lift of any loop representing $v$ and $\gamma(0)=\tilde{x}_{0}$.

Obviously, $\mathfrak{a}$ takes the powers of the distinguished element to points in $\mathcal{I}$ and takes the elements in $\check{\pi}$ to points in the interval $\mathcal{J}$. Thus, the composition of $\mathfrak{a}$ and the homeomorphism $j: \mathcal{J} \rightarrow \mathbb{R}$ is well defined on the set $\check{\pi}$ : we put

$$
\mathfrak{b}:=j \circ \mathfrak{a}: \check{\pi} \rightarrow \mathbb{R} .
$$

Under the embedding $\mathfrak{b}$, the usual ordering on $\mathbb{R}$ induces a linear ordering on $\check{\pi}$ (we denote it by $\leq$ ).

Remark. The following properties are obvious.

1. The ordering $\leq$ on the set $\check{\pi}$ is canonical: it is completely determined by the choice of the orientation on $\partial D_{n}$. (In particular, this ordering does not depend on the choice of the base point and the hyperbolic structure.)

2. The action of $B_{n} \cong \operatorname{MCG}\left(D_{n}\right)$ on $\check{\pi}$ preserves this canonical ordering.

4.3. Proposition. Suppose that $\beta \in B_{n}, g \in \operatorname{MCG}\left(D_{n}\right)$ is the corresponding mapping class, and $v \in \check{\pi}$ is any element. Then

$$
\omega(\beta)=\lim _{s \rightarrow \infty} \frac{\max \left\{k \in \mathbb{Z} \mid u^{k} v \leq g^{s}(v)\right\}}{s} .
$$

Proof. The definitions of the Nielsen-Thurston action and of the injection $\mathfrak{b}$ imply that for any $v \in \check{\pi}$ and $g \in \operatorname{MCG}\left(D_{n}\right)$ we have $(\widetilde{\Omega}(g))(\mathfrak{b}(v))=\mathfrak{b}(g v)$. By the definitions of $u$ and $\Theta \mid \mathcal{u}$, for each $v \in \check{\pi}$ we have $\mathfrak{a}(u v)=\Theta(\mathfrak{a}(v))$, i.e., $\mathfrak{b}(u v)=\mathfrak{b}(v)+1$. It follows that the inequality $u^{k} v \leq g^{s}(v)$ is equivalent to $\mathfrak{b}(v)+k \leq\left(\widetilde{\Omega}\left(g^{s}\right)\right)(\mathfrak{b}(v))$. Denoting by $a$ the point $\mathfrak{b}(v) \in \mathbb{R}$, we obtain

$$
\begin{aligned}
\max & \left\{k \in \mathbb{Z} \mid u^{k} v \leq g^{s}(v)\right\} \\
& =\max \left\{k \in \mathbb{Z} \mid a+k \leq\left(\widetilde{\Omega}\left(\beta^{s}\right)\right)(a)\right\}=\left[\left(\widetilde{\Omega}\left(\beta^{s}\right)\right)(a)-a\right],
\end{aligned}
$$

whence

$$
\begin{aligned}
\lim _{s \rightarrow \infty} & \frac{\max \left\{k \in \mathbb{Z} \mid u^{k} v \leq g^{s}(v)\right\}}{s} \\
& =\lim _{s \rightarrow \infty} \frac{\left[\left(\widetilde{\Omega}\left(g^{s}\right)\right)(a)-a\right]}{s}=\lim _{s \rightarrow \infty} \frac{\left(\widetilde{\Omega}\left(g^{s}\right)\right)(a)}{s}=\omega(g) .
\end{aligned}
$$




\section{§5. Properties of The TWIST NUMbeR}

Here we prove some basic properties of the twist number.

5.1. Lemma. We have $\omega\left(\Delta^{2}\right)=1$.

Proof. As before, $D_{n}$ is the punctured disk. Consider an isotopy $h_{t}: D_{n} \rightarrow D_{n}$ of $D_{n}$ $\left(t \in[0,1], h_{0}=\operatorname{id}_{D_{n}}\right)$ that is identical outside a neighborhood of the boundary $\partial D_{n}$ and performs a full turn of $\partial D_{n}$ in the counterclockwise direction: each point of $\partial D_{n}$ is taken to itself after passing once along the entire circle $\partial D_{n}$. The class $T \in \operatorname{MCG}\left(D_{n}\right)$ of the autohomeomorphism $h_{1} \in \mathrm{Homeo}_{+}\left(D_{n}, \partial D_{n}\right)$ is called the boundary Dehn twist. It is well known that the canonical isomorphism $B_{n} \cong \operatorname{MCG}\left(D_{n}\right)$ takes the element $\Delta^{2} \in B_{n}$ to $T$. Thus, by definition, we have $\omega\left(\Delta^{2}\right)=\omega(T)$.

We let $h_{t}^{\tilde{t}}: \mathcal{U} \rightarrow \mathcal{U}$ be the lifting of the isotopy $h_{t}$ (i.e., $h_{0}^{\sim}=\operatorname{id}_{\mathcal{U}}$ ). The isotopy $h_{t}^{\sim}$ shifts each point of the axis $\mathcal{I}$ in the negative direction (see Subsection 3.3) through a distance equal to the length of $\partial D_{n}$. Consequently, the autohomeomorphism $\phi^{\sim}:=\Theta h_{1}^{\sim}$ is identical on $\mathcal{I}$. Since, obviously, $\phi^{\sim}$ is a lift of $h_{1} \in T$, it follows (by definition) that $\Omega(T)=\left.\phi^{\bar{\sim}}\right|_{\mathcal{J}}$. Now, we observe that the isotopy $h_{t}^{\sim}$ shifts points through a bounded hyperbolic distance; hence, the extension of $h_{t}^{\sim}$ to $\mathcal{D}$ is identical at the points of the Cantor set $\mathcal{K}$ : we have $\left.h_{1}^{\overline{1}}\right|_{\mathcal{K}} \equiv \operatorname{id}_{\mathcal{K}}$. Thus,

$$
\left.\left.\left.\Omega(T)\right|_{\mathcal{K}} \equiv \Theta h_{1}^{\overline{1}}\right|_{\mathcal{K}} \equiv \Theta\right|_{\mathcal{K}}
$$

By definition, we have $\omega(T)=\lim _{s \rightarrow \infty} \frac{(\widetilde{\Omega}(T))^{s}(y)}{s}$ for any $y \in \mathbb{R}$. Take $y \in j(\mathcal{K} \cap \mathcal{J})$ (the set $\mathcal{K} \cap \mathcal{J}$ is $\mathcal{K}$ without two limit points of the interval $\mathcal{I}$ ). Then

$$
(\widetilde{\Omega}(T))(y)=\left(j_{*} \Theta\right)(y)=t_{1}(y)=y+1 .
$$

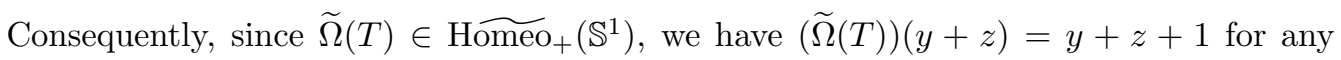
$z \in \mathbb{Z}$. It follows that

$$
\omega\left(\Delta^{2}\right)=\omega(T)=\lim _{s \rightarrow \infty} \frac{y+s}{s}=1 .
$$

5.2. Lemma (Property S). For any $\beta_{1}, \beta_{2} \in B_{n}$ and any $i \in\{1, \ldots, n-1\}$, we have

$$
\omega\left(\beta_{1} \beta_{2}\right) \leq \omega\left(\beta_{1} \sigma_{i} \beta_{2}\right) .
$$

Remark. Confer the "Property S" of Thurston-type orderings on the braid group, as defined in $[5]$.

Proof. We denote by $\beta a$ the image of a point $a \in \mathbb{R}$ under the action of an element $\widetilde{\Omega}(\beta)$. We recall that the autohomeomorphisms in $\widetilde{H}^{\text {omeo }}+\left(\mathbb{S}^{1}\right)$ preserve the orientation on $\mathbb{R}$; therefore,

$$
\forall \beta \in B_{n} ; a_{1}, a_{2} \in \mathbb{R}: \quad a_{1} \leq a_{2} \Longleftrightarrow \beta a_{1} \leq \beta a_{2} .
$$

It is well known that the braid $\sigma_{i}^{2}$ in $\operatorname{MCG}\left(D_{n}\right)$ corresponds to a Dehn twist along the corresponding curve. The Nielsen-Thurston action for Dehn twists in the general case of an oriented surface was considered in [14, Proposition 2.4]; in the case under consideration this proposition yields the following:

$$
\forall i \in\{1, \ldots, n-1\}, a \in \mathbb{R}: \quad \sigma_{i}^{2} a \geq a .
$$

From (5.2.1) and (5.2.2) we deduce that

$$
\forall i \in\{1, \ldots, n-1\}, a \in \mathbb{R}: \quad \sigma_{i} a \geq a,
$$

whence

$$
\beta_{1} \sigma_{i} \beta_{2} a=\beta_{1}\left(\sigma_{i}\left(\beta_{2} a\right)\right) \geq \beta_{1}\left(\beta_{2} a\right)=\beta_{1} \beta_{2} a .
$$


Using induction on $s \in \mathbb{N}$ and (5.2.1), (5.2.4), we obtain

$$
\left(\beta_{1} \sigma_{i} \beta_{2}\right)^{s} a \geq\left(\beta_{1} \beta_{2}\right)^{s} a .
$$

Now, the definition of the twist number (see Subsection 3.6) yields

$$
\omega\left(\beta_{1} \sigma_{i} \beta_{2}\right)=\lim _{s \rightarrow \infty} \frac{\left(\beta_{1} \sigma_{i} \beta_{2}\right)^{s} a}{s} \geq \lim _{s \rightarrow \infty} \frac{\left(\beta_{1} \beta_{2}\right)^{s} a}{s}=\omega\left(\beta_{1} \beta_{2}\right) .
$$

5.3. Definition. We say that a braid $\beta \in B_{n}$ is split if for some $r \in\{1, \ldots, n-1\}$ it is represented by a word in letters $\left\{\sigma_{1}^{ \pm 1}, \ldots, \sigma_{n-1}^{ \pm 1}\right\} \backslash\left\{\sigma_{r}^{ \pm 1}\right\}$.

5.4. Lemma on split braids. The twist number of any split braid is zero.

Proof. Suppose a braid $\beta \in B_{n}$ is represented by a word in letters $\left\{\sigma_{1}^{ \pm 1}, \ldots, \sigma_{n-1}^{ \pm 1}\right\} \backslash$ $\left\{\sigma_{r}^{ \pm 1}\right\}$. We prove that the autohomeomorphism $\widetilde{\Omega}(\beta)$ has a fixed point. We use the results and notation of $\S 2$ and Subsection 1.3. Let $g \in \operatorname{MCG}\left(D_{n}\right)$ be the mapping class corresponding to the braid $\beta$. Also, let $v \in \check{\pi}$ be the homotopy class of the loop with endpoints at $x_{0} \in \partial D_{n}$ that intersects the interval $(-1,1) \subset \mathbb{C}$ at precisely two points such that the first of them lies between the point $-1 \in \mathbb{C}$ and the first puncture, while the second lies between the punctures $r$ and $r+1$. Obviously, $g(v)=v$, so that (see the proof of Proposition 4.3) we have

$$
(\widetilde{\Omega}(g))(\mathfrak{b}(v))=\mathfrak{b}(g v)=\mathfrak{b}(v) .
$$

By the definition of the twist number, we obtain

$$
\omega(\beta)=\lim _{s \rightarrow \infty} \frac{(\widetilde{\Omega}(g))^{s}(\mathfrak{b}(v))}{s}=\lim _{s \rightarrow \infty} \frac{\mathfrak{b}(v)}{s}=0 .
$$

5.5. Corollary. Suppose a braid $\beta \in B_{n}$ is represented by a braid word $W$ that does not contain the letter $\sigma_{i}^{-1}$ for some $i \in\{1, \ldots, n-1\}$. Then $\omega(\beta) \geq 0$.

Proof. We denote by $V$ the braid word obtained by deleting all occurrences of the letter $\sigma_{i}$ from $W$, and let $\alpha \in B_{n}$ be the braid represented by $V$. Since $V$ is a word in the generators $\left\{\sigma_{1}^{ \pm 1}, \ldots, \sigma_{n-1}^{ \pm 1}\right\} \backslash\left\{\sigma_{i}^{ \pm 1}\right\}$, we see that the braid $\alpha$ is split, and Lemma 5.4 implies that $\omega(\alpha)=0$. It remains to observe that $\omega(\alpha) \leq \omega(\beta)$ by Lemma 5.2.

\section{$\S 6$. THE TWIST NUMBer AS A PSEUdOCHARACTER}

In this section, we show that the twist number is a pseudocharacter on the braid group, list the properties of the twist number as a pseudocharacter, and compute its defect.

6.1. Pseudocharacters. A functional $\varphi: G \rightarrow \mathbb{R}$ on a group $G$ is called a pseudocharacter (or pseudohomomorphism) if the following conditions are fulfilled:

$$
\begin{gathered}
\sup _{g_{1}, g_{2} \in G}\left|\varphi\left(g_{1} g_{2}\right)-\varphi\left(g_{1}\right)-\varphi\left(g_{2}\right)\right|=D_{\varphi}<\infty ; \\
\forall z \in \mathbb{Z}, g \in G: \quad \varphi\left(g^{z}\right)=z \varphi(g) .
\end{gathered}
$$

The number $D_{\varphi}$ is called the defect of $\varphi$.

6.2. Proposition (see, e.g., 6]). A pseudocharacter takes conjugate elements of the group to the same value. The restriction of a pseudocharacter to any Abelian subgroup of $G$ is a homomorphism.

6.3. Theorem. The twist number $\omega: B_{n} \rightarrow \mathbb{R}$ is a pseudocharacter on the group $B_{n}$. If $n>2$, then the defect of the pseudocharacter $\omega$ is equal to 1 . The pseudocharacter $\omega: B_{2} \rightarrow \mathbb{R}$ is the homomorphism $\sigma_{1}^{z} \mapsto z / 2$. 


\subsection{The properties of the twist number as a pseudocharacter.}

6.4.1. For any $\beta_{1}, \beta_{2} \in B_{n}$, we have

$$
\left|\omega\left(\beta_{1} \beta_{2}\right)-\omega\left(\beta_{1}\right)-\omega\left(\beta_{2}\right)\right| \leq 1 .
$$

6.4.2. For any $\beta_{1}, \beta_{2} \in B_{n}$ such that $\beta_{1} \beta_{2}=\beta_{2} \beta_{1}$, we have

$$
\omega\left(\beta_{1} \beta_{2}\right)=\omega\left(\beta_{1}\right)+\omega\left(\beta_{2}\right) .
$$

6.4.2'. For any $\beta \in B_{n}$ and $k, z \in \mathbb{Z}$, we have

$$
\omega\left(\beta^{k} \Delta^{2 z}\right)=k \omega(\beta)+z .
$$

6.4.3. The twist number is a conjugacy invariant, i.e., for any $\alpha, \beta \in B_{n}$ we have

$$
\omega\left(\alpha \beta \alpha^{-1}\right)=\omega(\beta) \text {. }
$$

Proof of Theorem 6.3 and of the properties given above. It is well known that the translation number is a pseudocharacter with defect equal to 1. (See, e.g., [7.) It is easily seen that the composition of a homomorphism and a pseudocharacter with defect $C$ is also a pseudocharacter with a defect at most $C$. Thus, by Definition 3.6, the twist number is a pseudocharacter with defect at most 1, i.e., the first assertion of the theorem and property 6.4 .1 are true.

Proposition 6.2 implies properties 6.4 .2 and 6.4.3, while property 6.4 .2 and Lemma 5.1 imply property $6.4 .2^{\prime}$. The final assertion of the theorem is obvious.

It remains to show that for $n>2$ the defect of the pseudocharacter $\omega: B_{n} \rightarrow \mathbb{R}$ equals 1. Since we have already seen that the defect is at most 1 , it suffices to find two braids $\beta_{1}, \beta_{2} \in B_{n}$ with $\omega\left(\beta_{1} \beta_{2}\right)-\omega\left(\beta_{1}\right)-\omega\left(\beta_{2}\right)=1$.

By the definition of the fundamental braid, we have $\Delta=V \sigma_{n-1} W$, where $V$ and $W$ are words in the generators $\sigma_{1}, \ldots, \sigma_{n-2}$. We set $\rho:=W V$. The braids $\sigma_{n-1}, \rho$, and $\rho^{2}$ are split, and Lemma 5.4 implies

$$
\omega\left(\sigma_{n-1}\right)=\omega(\rho)=\omega\left(\rho^{2}\right)=0 .
$$

Since the braid $\Delta^{2}$ commutes with any braid, we have

$$
\Delta^{2}=V \sigma_{n-1} W V \sigma_{n-1} W=W V \sigma_{n-1} W V \sigma_{n-1}=\rho \sigma_{n-1} \rho \sigma_{n-1}
$$

By Lemma 5.1, $\omega\left(\Delta^{2}\right)=1$. Applying property $6.4 .2\left(\Delta^{2}\right.$ and $\sigma_{n-1}$ commute), we obtain

$$
\omega\left(\rho \sigma_{n-1} \rho\right)=\omega\left(\Delta^{2}\right)-\omega\left(\sigma_{n-1}\right)=1-0=1 .
$$

The braid $\rho \sigma_{n-1} \rho$ is conjugate to $\rho^{2} \sigma_{n-1}$, whence $\omega\left(\rho^{2} \sigma_{n-1}\right)=\omega\left(\rho \sigma_{n-1} \rho\right)=1$. Thus,

$$
\omega\left(\rho^{2} \sigma_{n-1}\right)-\omega\left(\rho^{2}\right)-\omega\left(\sigma_{n-1}\right)=1-0-0=1 .
$$

\section{$\S 7$. DEHORNOY'S ORDERING}

In this section, we prove some relations between the twist number and Dehornoy's ordering.

7.1. Definition. A braid $\beta \in B_{n}$ is said to be $\sigma$-positive if for some $i \in\{1, \ldots, n-1\}$ it is represented by a braid word of the form $V \sigma_{i}^{+1} W$, where $V$ and $W$ are words in letters $\left\{\sigma_{i}^{+1}, \sigma_{i+1}^{ \pm 1}, \ldots, \sigma_{n-1}^{ \pm 1}\right\}$. Suppose $\beta_{1}, \beta_{2} \in B_{n}$. We write $\beta_{1} \prec \beta_{2}$ if the braid $\beta_{2} \beta_{1}^{-1}$ is $\sigma$-positive. The relation $\prec$ is a right-invariant linear ordering on the braid group [5], i.e.,

$$
\forall \beta_{1}, \beta_{2}, \alpha \in B_{n}: \quad \beta_{1} \prec \beta_{2} \Longleftrightarrow \beta_{1} \alpha \prec \beta_{2} \alpha,
$$

and we call it Dehornoy's ordering. 
7.2. Lemma. 1) If $\beta$ is a $\sigma$-positive braid, then $\omega(\beta) \geq 0$.

2) If two braids $\beta_{1}$ and $\beta_{2}$ commute, then the following two implications are true:

$$
\omega\left(\beta_{1}\right)<\omega\left(\beta_{2}\right) \underset{\text { a) }}{\Longrightarrow} \beta_{1} \prec \beta_{2} \underset{\mathrm{b})}{\Longrightarrow} \omega\left(\beta_{1}\right) \leq \omega\left(\beta_{2}\right) .
$$

3) If $\beta \in B_{n}$ and $a<b \in \mathbb{Z}$, then

$$
a<\omega(\beta)<b \Longrightarrow \Delta^{2 a} \prec \beta \prec \Delta^{2 b} \Longrightarrow a \leq \omega(\beta) \leq b .
$$

Proof. Statement 1) follows from Corollary 5.5.

2b) By the definition of Dehornoy's ordering, the condition $\beta_{1} \prec \beta_{2}$ means that the braid $\beta_{2} \beta_{1}^{-1}$ is $\sigma$-positive. In this case, we have $\omega\left(\beta_{2} \beta_{1}^{-1}\right) \geq 0$ by assertion 1$)$. Since $\beta_{1}$ and $\beta_{2}$ commute, Property 6.4.2 yields $\omega\left(\beta_{2} \beta_{1}^{-1}\right)=\omega\left(\beta_{2}\right)-\omega\left(\beta_{1}\right)$, whence $\omega\left(\beta_{2}\right) \geq \omega\left(\beta_{1}\right)$.

2a) Suppose $\beta_{1} \nprec \beta_{2}$. If $\beta_{1}=\beta_{2}$, then $\omega\left(\beta_{1}\right)=\omega\left(\beta_{2}\right)$. If $\beta_{2} \prec \beta_{1}$, then $\omega\left(\beta_{1}\right) \geq \omega\left(\beta_{2}\right)$ by the implication $2 \mathrm{~b})$, a contradiction.

3) We have $\omega\left(\Delta^{2 a}\right)=a$ and $\omega\left(\Delta^{2 b}\right)=b$. Also, observe that the braids $\Delta^{2 a}$ and $\Delta^{2 b}$ commute with $\beta$. It remains to apply part 2 ).

7.3. Definition. Dehornoy floor. It is well known that for each braid $\beta$ there is an (obviously, unique) $z \in \mathbb{Z}$ such that

$$
\Delta^{2 z} \preceq \beta \prec \Delta^{2(z+1)}
$$

(see, e.g., [13]). We denote $z$ by $[\beta]_{D}$ and call it the Dehornoy floor (or the integral part) of $\beta$.

7.4. Lemma. For each braid $\beta$, we have

$$
[\beta]_{D} \leq \omega(\beta) \leq[\beta]_{D}+1 .
$$

Proof. This immediately follows from assertion 3 of Lemma 7.2 and the definition of the Dehornoy floor.

7.5. Theorem. For each braid $\beta$, we have

$$
\omega(\beta)=\lim _{s \rightarrow \infty} \frac{\left[\beta^{s}\right]_{D}}{s} .
$$

Proof. Lemma 7.4 implies that

$$
0 \leq \omega(\alpha)-[\alpha]_{D} \leq 1
$$

for each braid $\alpha$. Therefore, since the twist number is a pseudocharacter and $s \omega(\beta)=$ $\omega\left(\beta^{s}\right)$, we have

$$
\left|\omega(\beta)-\frac{\left[\beta^{s}\right]_{D}}{s}\right|=\left|\frac{\omega\left(\beta^{s}\right)-\left[\beta^{s}\right]_{D}}{s}\right| \leq \frac{1}{s} .
$$

The latter inequality implies the assertion of the theorem.

7.6. Remark. A functional $\psi: G \rightarrow \mathbb{R}$ on a group $G$ is called a quasicharacter (or a quasihomomorphism) with defect $C$ if the following condition is fulfilled:

$$
\sup _{g_{1}, g_{2} \in G}\left|\psi\left(g_{1} g_{2}\right)-\psi\left(g_{1}\right)-\psi\left(g_{2}\right)\right|=C<\infty .
$$

It is well known (see, e.g., [6]) that if $\psi: G \rightarrow \mathbb{R}$ is a quasicharacter, then for each $g \in G$ the sequence $\left\{\psi\left(g^{s}\right) / s\right\}$ has a finite limit, and the functional

$$
\varphi: G \rightarrow \mathbb{R}, \quad \varphi(g):=\lim _{s \rightarrow \infty} \frac{\psi\left(g^{s}\right)}{s}
$$

is a pseudocharacter.

Thus, the Dehornoy floor is a quasicharacter on the braid group (it can be shown that its defect is equal to 1), and the twist number is the corresponding pseudocharacter. 


\section{§8. UNIQUENESS THEOREM}

8.1. Theorem. There exists a unique pseudocharacter $B_{n} \rightarrow \mathbb{R}$ that takes a nonnegative value on each $\sigma$-positive braid and takes value 1 on the braid $\Delta^{2}$.

Remark. The above theorem admits numerous reformulations.

For example, the theorem remains valid (and the twist number remains to be a pseudocharacter satisfying the assumptions of the theorem) if we replace the $\sigma$-positivity in its formulation by the positivity with respect to any other Thurston-type ordering on the braid group.

With the same result, we can replace the requirement of taking nonnegative values "on the $\sigma$-positive braids" by the stronger requirement of taking a nonnegative value "on each braid represented by a word not containing the generator $\sigma_{1}^{-1}$ ", in which way we exclude mentioning the ordering in the formulation.

Furthermore, the twist number is a unique pseudocharacter on the braid group that possesses the properties mentioned in Lemmas 5.1, 5.2, and 5.4.

Proof. Existence. The twist number satisfies all conditions of the theorem. Indeed, by Theorem 6.3, the twist number is a pseudocharacter. By part 1 of Lemma 7.2, we have $\omega(\beta) \geq 0$ whenever $\beta$ is $\sigma$-positive. Finally, $\omega\left(\Delta^{2}\right)=1$ (Lemma 5.1).

Uniqueness. Suppose $\omega^{\prime}$ is a pseudocharacter satisfying the conditions of the theorem. Recalling $\$ 7$, we observe that the successive proof of assertions 2 and 3 of Lemma 7.2, Lemma 7.4, and Theorem 7.5 involves only the following facts:

a) the twist number is a pseudocharacter,

b) $\omega\left(\Delta^{2}\right)=1$, and

c) the twist number takes nonnegative values on $\sigma$-positive braids (assertion 1 of Lemma 7.2).

At the same time, by assumption we have the following:

$\left.\mathrm{a}^{\prime}\right) \omega^{\prime}$ is a pseudocharacter,

$\left.\mathrm{b}^{\prime}\right) \omega^{\prime}\left(\Delta^{2}\right)=1$, and

$\left.\mathrm{c}^{\prime}\right)$ the pseudocharacter $\omega^{\prime}$ takes nonnegative values on $\sigma$-positive braids.

Therefore, repeating for $\omega^{\prime}$ the chain of proofs in assertions 2) and 3) of Lemma 7.2, Lemma 7.4, and Theorem 7.5 "word for word", we see that for each braid $\beta$ we have the identity

$$
\omega^{\prime}(\beta)=\lim _{s \rightarrow \infty} \frac{\left[\beta^{s}\right]_{D}}{s} .
$$

Applying Theorem 7.5, we obtain $\omega^{\prime} \equiv \omega$.

\section{$\S 9$. TWIST NUMBER OF A CLOSED BRAID}

9.1. Closed braids. Suppose $\mathcal{L}$ is an oriented link type in the oriented 3 -sphere $S^{3}$ (or in $\left.\mathbb{R}^{3}=S^{3} \backslash\{\infty\}\right)$. Suppose $L \in \mathcal{L}$ is a representative of $\mathcal{L}$, and $A \subset S^{3} \backslash L$ a curve unknotted in $S^{3}$.

The link $L$ is called a closed braid with axis $A$ if there is a fibration $H=\left\{H_{\theta} ; \theta \in\right.$ $[0,2 \pi]\}$ of the open solid torus $S^{3} \backslash A$ into meridional disks $H_{\theta}$ such that $L$ intersects each disk $H_{\theta}$ transversally. The number of points of intersection of $L$ and $H_{\theta}$ is called the index of the closed braid $L$ (it does not depend on the choice of $\theta$ ). We shall use the term "closed braid" to refer to an isotopy class of closed braids as well.

The standard "closing procedure" (see, e.g., [1]) transforms a braid $\beta \in B_{n}$ into a closed braid $\widehat{\beta}$ of index $n$. It is well known that there is a one-to-one correspondence between the conjugacy classes in the braid groups $B_{1}, B_{2}, B_{3}, \ldots$ and the isotopy classes of closed braids. For this reason, if $\beta$ is a braid, then we denote by $\widehat{\beta}$ both the conjugacy class of $\beta$ and the corresponding closed braid, which we identify with the conjugacy class. 
9.2. Definition. The twist number is a conjugacy invariant (since it is a pseudocharacter), and thus the twist number of a closed braid is well defined: we put $\omega(\widehat{\beta}):=\omega(\beta)$.

\section{$\S 10$. Satellite BRAids}

10.1. Definition. A closed braid $\widehat{\beta}$ is a satellite of a closed braid $\widehat{\alpha}$ if the index of the braid $\widehat{\alpha}$ is greater than 1 and there exist a collection of solid tori $T \subset \mathbb{R}^{3}$, a link $L \subset \operatorname{int} T$, and an axis $A \subset \mathbb{R}^{3} \backslash T$ such that

- $L$ represents the closed braid $\widehat{\beta}$ with respect to the axis $A$;

- the axes of the solid tori in $T$ (oriented in accordance with the orientation of $L$ ) represent the closed braid $\widehat{\alpha}$ with respect to the axis $A$; and

- each component of $T$ contains at least one component of $L$.

10.2. Theorem. Suppose a closed braid $\widehat{\beta}$ is a satellite of a closed braid $\widehat{\alpha}$. Then $\omega(\widehat{\beta})=\omega(\widehat{\alpha})$.

Proof. Let $A, L$, and $T$ be as in Definition 10.1. Obviously, we can assume without loss of generality that $L$ and $T$ lie inside some closed solid torus $V \subset S^{3} \backslash A$, and that there is a fibration $H=\left\{H_{\theta} ; \theta \in[0,2 \pi]\right\}$ of the solid torus $V$ into meridional disks $H_{\theta}$ such that each of them transversally intersects the link $L$ and the collection of tori $\partial T$.

We denote by $m$ and $n$ the indices of the braids $\widehat{\alpha}$ and $\widehat{\beta}$, respectively. Then each disk $H_{\theta}$ intersects $T$ along $m$ disks. We denote by $D$ the disk $H_{0}=H_{2 \pi}$, by $D_{n}:=D \backslash L$ the disk with $n$ punctures, and by $D_{m}:=D \backslash T$ the disk with $m$ disks cut off. (Clearly, $D_{m}$ is homeomorphic to a disk with $m$ punctures.)

The above description of the triples $L \subset T \subset V$ and $D_{m} \subset D_{n} \subset D$ shows that there is an autohomeomorphism $\phi: D \rightarrow D$ identical on $\partial D$ and such that the following is true (for the corresponding orientation of $D$ ):

- $\phi\left(D_{n}\right)=D_{n}$, and the class of the autohomeomorphism $\left.\phi\right|_{D_{n}}$ corresponds to the braid $\widehat{\beta}$;

- $\phi\left(D_{m}\right)=D_{m}$, and the class of the autohomeomorphism $\left.\phi\right|_{D_{m}}$ corresponds to the braid $\widehat{\alpha}$.

Choose a point $x_{0} \in \partial D=\partial D_{n}=\partial D_{m}$ and consider the groups $\pi_{1}\left(D_{m}, x_{0}\right)$ and $\pi_{1}\left(D_{n}, x_{0}\right)$. Here, we use the construction and notation of $\S 4$.

Since each disk in $D \cap T$ contains at least one point of $D \cap L$ (this directly follows from the definition of a satellite braid), the natural homomorphism

$$
i_{\pi}: \pi_{1}\left(D_{m}, x_{0}\right) \rightarrow \pi_{1}\left(D_{n}, x_{0}\right)
$$

is a monomorphism. We denote by $\phi_{1}$ (respectively, $\left.\phi_{2}\right)$ the automorphism of $\pi_{1}\left(D_{m}, x_{0}\right)$ (respectively, $\left.\pi_{1}\left(D_{n}, x_{0}\right)\right)$ induced by the autohomeomorphism $\left.\phi\right|_{D_{m}}$ (respectively, $\left.\phi\right|_{D_{n}}$ ). Furthermore, we obviously have

$$
i_{\pi} \circ \phi_{1} \equiv \phi_{2} \circ i_{\pi} .
$$

Letting $u_{1} \in \pi_{1}\left(D_{m}, x_{0}\right)$ and $u_{2} \in \pi_{1}\left(D_{n}, x_{0}\right)$ be the distinguished elements, we denote by $\leq_{1}$ and $\leq_{2}$ the canonical orderings on $\check{\pi}_{1}\left(D_{m}, x_{0}\right)$ and $\check{\pi}_{1}\left(D_{n}, x_{0}\right)$, respectively. Then, obviously,

$$
i_{\pi}\left(u_{1}\right)=u_{2},
$$

and $i_{\pi}$ takes the ordering $\leq_{1}$ to $\leq_{2}$ (see the definition of the canonical ordering in $\S 4$ ), i.e.,

$$
\forall v, w \in \check{\pi}_{1}\left(D_{m}, x_{0}\right): \quad v \leq_{1} w \Longleftrightarrow i_{\pi}(v) \leq_{2} i_{\pi}(w) .
$$


Let $v_{1} \in \check{\pi}_{1}\left(D_{m}, x_{0}\right)$ be any element, and let $v_{2}:=i_{\pi}\left(v_{1}\right)$. From (10.2.1)-(10.2.3) it follows that for any $k \in \mathbb{Z}$ and $s \in \mathbb{N}$ we have

$$
u_{1}^{k} v_{1} \leq_{1} \phi_{1}^{s}\left(v_{1}\right) \Longleftrightarrow i_{\pi}\left(u_{1}^{k} v_{1}\right) \leq_{2} i_{\pi}\left(\phi_{1}^{s}\left(v_{1}\right)\right) \Longleftrightarrow u_{2}^{k} v_{2} \leq_{2} \phi_{2}^{s}\left(v_{2}\right) .
$$

Applying the formula of Proposition 4.3, we obtain

$$
\begin{aligned}
\omega(\widehat{\alpha}) & =\omega\left(\left[\left.\phi\right|_{D_{m}}\right]\right)=\lim _{s \rightarrow \infty} \frac{\max \left\{k \in \mathbb{Z} \mid u_{1}^{k} v_{1} \leq_{1} \phi_{1}^{s}\left(v_{1}\right)\right\}}{s} \\
& =\lim _{s \rightarrow \infty} \frac{\max \left\{k \in \mathbb{Z} \mid u_{2}^{k} v_{2} \leq_{2} \phi_{2}^{s}\left(v_{2}\right)\right\}}{s}=\omega\left(\left[\left.\phi\right|_{D_{n}}\right]\right)=\omega(\widehat{\beta}) .
\end{aligned}
$$

10.3. Remark. Since each split braid is a satellite of a trivial braid of index 2 , we can regard Lemma 5.4 as a special case of Theorem 10.2.

\section{§11. Rationality}

Our main purpose in this section is the proof of the following result.

11.1. Theorem. The twist number of any braid is rational. Moreover,

$$
\omega\left(B_{n}\right)=\mathbb{Q}_{[n]}, \quad \text { where } \mathbb{Q}_{[n]}:=\left\{\frac{p}{q} \mid p \in \mathbb{Z}, n \geq q \in \mathbb{N}\right\} .
$$

The proof involves the Nielsen-Thurston classification of the surface automorphisms. It reveals the relationship between the twist number and certain elements of the NielsenThurston theory. By using the constructions presented below, the twist number can easily be described in the framework of this theory (cf. Menasco's definitions [10]).

11.2. Nielsen-Thurston classification for braids. We recall the definitions of types of braids in the Nielsen-Thurston classification.

In addition to the mapping class group $\operatorname{MCG}\left(D_{n}\right)$ defined above, we consider the mapping class group

$$
\operatorname{mcg}\left(D_{n}\right):=\text { Homeo }_{+}\left(D_{n}\right) / \operatorname{Homeo}_{0}\left(D_{n}\right),
$$

where $\mathrm{Homeo}_{+}\left(D_{n}\right)$ is the group of all orientation-preserving homeomorphisms of $D_{n}$, while $\operatorname{Homeo}_{0}\left(D_{n}\right)$ is the normal subgroup consisting of all homeomorphisms isotopic to the identity (cf. the definition of $\operatorname{MCG}\left(D_{n}\right)$ ).

Thurston's classification theorem states that each class $h \in \operatorname{mcg}\left(D_{n}\right)$ belongs to one of the following three types.

Periodic: The class $h$ has finite order (i.e., $h^{q}=e$ for some $q \in \mathbb{N}$ ).

Pseudo-Anosov: In this case, for the hyperbolic metric on $D_{n}$ introduced above (see $\S 2$ ), there is an autohomeomorphism $\psi \in h$ and a pair of mutually transverse geodesic laminations $L_{s}$ and $L_{u}$ (stable and unstable) possessing certain properties and such that $\psi\left(L_{s}\right)=L_{s}$ and $\psi\left(L_{u}\right)=L_{u}$ (see [16, 15]).

Reducible: The class $h$ contains an autohomeomorphism preserving some closed onedimensional nonempty submanifold $C \subset D_{n}$, no component of which is contractible to a point or to $\partial D_{n}$, and, furthermore, the components of $C$ are nonisotopic. In this case, in each (possibly, disconnected) component of the surface $D_{n} \backslash C$ the induced autohomeomorphism belongs either to the periodic, or to the pseudo-Anosov type.

It is well known that the natural embedding

$$
\mathrm{Homeo}_{+}\left(D_{n}, \partial D_{n}\right) \rightarrow \mathrm{Homeo}_{+}\left(D_{n}\right)
$$

induces an epimorphism

$$
\mathrm{mc}: B_{n} \cong \operatorname{MCG}\left(D_{n}\right) \rightarrow \operatorname{mcg}\left(D_{n}\right) \text {. }
$$


The kernel of mc is the infinite cyclic subgroup generated by the element $\Delta^{2} \in B_{n}$. We have the following exact sequence (cf. the first row of the commutative diagram in Subsection 3.1):

$$
0 \rightarrow \mathbb{Z} \rightarrow B_{n} \rightarrow \operatorname{mcg}\left(D_{n}\right) \rightarrow 1 .
$$

The type of a braid $\beta$ is defined to be that of the class $\operatorname{mc}(\beta) \in \operatorname{mcg}\left(D_{n}\right)$.

11.3. Now we can pass to the proof of Theorem 11.1, which consists in verification of two inclusions:

$$
\omega\left(B_{n}\right) \subset \mathbb{Q}_{[n]} \text { and } \omega\left(B_{n}\right) \supset \mathbb{Q}_{[n]} .
$$

11.4. Assertion. We have $\omega\left(B_{n}\right) \subset \mathbb{Q}_{[n]}$.

Proof. Suppose $\beta \in B_{n}$ is a braid and $g \in \operatorname{MCG}\left(D_{n}\right)$ is the corresponding mapping class. By the Thurston theorem, $\beta$ is of periodic, pseudo-Anosov, or reducible type. We consider each case separately.

11.4.1. Periodic type. Suppose that $\beta$ is a braid of periodic type, i.e., the element $\operatorname{mc}(\beta) \in \operatorname{mcg}\left(D_{n}\right)$ is periodic, say, of order $q \in \mathbb{N}$, which means that $\beta^{q} \in$ ker mc. Since ker mc is generated by $\Delta^{2}$, we have $\beta^{q}=\Delta^{2 p}$ for some $p \in \mathbb{Z}$, whence by property $6.4 .2^{\prime}$ it follows that $\omega(\beta)=p / q \in \mathbb{Q}$.

Now we analyze which values $q$ can take. By the Nielsen realization theorem, the class $\operatorname{mc}(\beta)$ contains a periodic autohomeomorphism $\psi: D_{n} \rightarrow D_{n}$ (i.e., $\psi^{q} \equiv$ id). We denote by $\bar{D}_{n}$ an $n$-point compactification of $D_{n}$, i.e., $\bar{D}_{n}$ is the unit disk $D^{2}$ with $n$ distinguished points. Then, obviously, $\psi$ extends to a periodic autohomeomorphism $\bar{\psi}: \bar{D}_{n} \rightarrow \bar{D}_{n}$ of order $q$ preserving the set of distinguished points.

By the Kerékjártó theorem (see [4, 9, 2]), each orientation-preserving periodic autohomeomorphism of the disk is conjugate to a Euclidean rotation. We do not consider the "trivial" case where $q=1$ : then $\operatorname{mc}(\beta)$ is a trivial element, and $\bar{\psi}$ is an identical mapping. If $q \geq 2$, then $\bar{\psi}$ has a fixed point $O \in \bar{D}_{n}$, the "center of the rotation". Obviously, there are two possibilities:

i) none of the distinguished points coincides with $O$;

ii) $O$ is one of the distinguished points.

The autohomeomorphism $\bar{\psi}$ acts on the set of distinguished points, splitting it into orbits. Since $\bar{\psi}$ is conjugate to a rotation, in case i) each orbit consists of $q$ elements; hence, $q$ divides $n$. In case ii), the orbit of $O$ is a singleton, while each of the other orbits, as before, consists of $q$ elements, so that $q$ divides $n-1$. Thus, $q$ divides either $n$ or $n-1$, whence $\omega(\beta)=p / q \in \mathbb{Q}_{[n]}$.

11.4.2. Pseudo-Anosov type. When considering this case, we use the notions and results of the theory of geodesic laminations (see [16, 15]), assuming that $D_{n}$ is equipped with a hyperbolic structure (see $\S 2$ ).

So, suppose that the braid $\beta$ is pseudo-Anosov, i.e., $\operatorname{mc}(\beta)$ is a class of pseudo-Anosov type. We denote by $L$ one of two invariant laminations for the class $\operatorname{mc}(\beta)$. We recall that $L$ is a closed subset of $D_{n}$ and is a union of disjoint simple geodesics. These geodesics are the leaves of $L$, and the connected components of the set $D_{n} \backslash L$ are the domains of the lamination $L$. The leaves adjoining a certain domain are called the boundary leaves of this domain.

Consider the domain of $L$ containing the circle $\partial D_{n}$; we denote this domain by $U$. It is well known that the interior of $U$ (i.e., the set $U \backslash \partial D_{n}$ ) is isometric to the interior of a certain crown. (A crown is a complete hyperbolic surface having finite area and geodesic boundary and homeomorphic to the space $\left(S^{1} \times[0,1]\right) \backslash V$, where $V$ is a finite subset of the circle $S^{1} \times\{1\}$.) As before, $\mathcal{U}$ denotes a universal covering space of $D_{n}$. We let $U^{\sim} \subset \mathcal{U}$ be the connected component of the preimage of $U$ in $\mathcal{U}$ that contains a geodesic 
$\mathcal{I}$ (see the notation in $\S \S 2$ and 3 ). The restriction of the covering map $\mathcal{U} \rightarrow D_{n}$ to $U^{\sim}$ is a universal covering of $U$, and the deck transformation $\left.\Theta\right|_{U \sim}$ generates a cyclic group of deck transformations of the latter. Factorizing the space $\operatorname{clos}\left(U^{\sim}\right)$, which is the closure of $U^{\sim}$ in $\mathcal{U}$, by the orbits of the action $\Theta$, we obtain a crown with interior isometric to the interior of $U$.

Claim. There exists an autohomeomorphism $\phi \in g$ such that $\phi(L)=L$.

(We recall that $g \in \operatorname{MCG}\left(D_{n}\right)$ is the mapping class corresponding to the braid $\beta$.)

Proof. By the Thurston theorem, the class $\operatorname{mc}(\beta)$ contains autohomeomorphisms preserving $L$. Let $\psi \in \operatorname{mc}(g)$ satisfy $\psi(L)=L$. We observe that the lamination $L$ does not intersect some tubular neighborhood $A$ of the boundary $\partial D_{n}$, so that $L \subset D_{n} \backslash A$. Obviously, there is an autohomeomorphism $\phi \in g$ with $\left.\left.\phi\right|_{D_{n} \backslash A} \equiv \psi\right|_{D_{n} \backslash A}$, whence $\phi(L)=\psi(L)=L$.

In what follows, we fix such an autohomeomorphism $\phi$.

As in $\S 2$, we denote by $\phi^{\sim}: \mathcal{U} \rightarrow \mathcal{U}$ the lifting of $\phi$ that is identical on $\mathcal{I}$, and by $\phi^{\bar{\sim}}$ the extension of $\phi^{\sim}$ to $\mathcal{D}$.

We observe that $\phi$ preserves the domain $U$ and cyclically permutes the boundary leaves of $U$. (Also, it is easily seen that $\phi^{\sim}\left(\operatorname{clos}\left(U^{\sim}\right)\right)=\operatorname{clos}\left(U^{\sim}\right)$.) We denote by $q$ the number of the boundary leaves in $U$. Then the autohomeomorphism $\phi^{q}$ preserves each of these leaves (and, obviously, preserves their orientations). Let $\ell \subset L$ be a boundary leaf of the domain $U$, and let $\ell_{1} \subset \operatorname{clos}\left(U^{\sim}\right)$ be one of the geodesics covering the leaf $\ell$ and lying on the boundary of the component $U^{\sim}$. Then the geodesic $\ell_{2}:=\left(\phi^{\sim}\right)^{q}\left(\ell_{1}\right) \subset \operatorname{clos}\left(U^{\sim}\right)$ also covers $\ell$. Hence, $\Theta^{p}\left(\ell_{1}\right)=\ell_{2}$ for some $p \in \mathbb{Z}$. (Each geodesic in $\operatorname{clos}\left(U^{\sim}\right)$ that covers $\ell$ is mapped to $\ell_{1}$ by a certain deck transformation of the covering $U^{\sim} \rightarrow U$, while the element $\left.\Theta\right|_{U \sim}$ generates the group of deck transformations of this covering.) We denote by $y \in \mathcal{J}$ any of the two limit points of $\ell_{1}$ on the absolute. By construction, we have $\left(\phi^{\widetilde{\sim}}\right)^{q} y=\Theta^{p} y$, i.e., $\left(\widetilde{\Omega}\left(\beta^{q}\right)\right) y=y+p$. Since $\widetilde{\Omega}\left(\beta^{q}\right) \in \mathrm{Homeo}_{+}\left(\mathbb{S}^{1}\right)$, it follows that $\left(\widetilde{\Omega}\left(\beta^{q}\right)\right)(y+z)=y+z+p$ for each integer $z$. Recalling the definition of the twist number, we see that $\omega\left(\beta^{q}\right)=p$, and we have $\omega(\beta)=p / q$ by property $6.4 .2^{\prime}$.

We show that the number $q$ of the boundary leaves of $U$ is at most $n-2$. It is well known that the hyperbolic area of a disk with $n$ punctures equals $2 \pi(n-1)$ (we recall that $D_{n}$ is equipped with a hyperbolic metric such that the boundary $\partial D_{n}$ is geodesic and the punctures are cusps; see Subsection 2.1). The area of the crown $U$ with $q$ "prongs" equals $q \pi$. Each puncture is contained in a certain domain of the lamination $L$, different from $U$ ( $U$ already contains the boundary, and so cannot contain any punctures), and it is known that each domain contains at most one puncture. The area of any domain is at least $\pi$, and the number of punctures is $n$; thus, the sum of the areas of the domains containing the punctures is at least $n \pi$. Therefore, $q \pi+n \pi \leq 2(n-1) \pi$, whence $q \leq n-2$.

Thus, in the pseudo-Anosov case we have $\omega(\beta)=p / q \in \mathbb{Q}_{[n-2]} \subset \mathbb{Q}_{[n]}$.

11.4.3. Reducible type. This case reduces to the two previous ones, because each reducible braid is a satellite of a certain pseudo-Anosov or periodic braid.

Indeed, suppose that $\beta$ is reducible and $C \subset D_{n}$ is a nonempty one-dimensional submanifold satisfying the requirements of the definition of a reducible class. (See Subsection 11.2: none of the components of $C$ is contractible either to a point or to $\partial D_{n} ; C$ is preserved by a certain autohomeomorphism in the class $\operatorname{mc}(\beta)$; and on each (possibly, disconnected) component of the cut surface $D_{n} \backslash C$ the induced autohomeomorphism belongs either to the periodic or to the pseudo-Anosov type.)

We denote by $M$ the component of $D_{n} \backslash C$ containing $\partial D_{n}$. The surface $M$ is homeomorphic to a disk with $m$ punctures, because $M$ is obtained from the punctured disk 
$D_{n}$ by cutting out several closed disks. Furthermore, we have $m<n$, because each of the cut-out disks contains at least two punctures of the disk $D_{n}$.

Arguing as in the pseudo-Anosov case, we see that there is an autohomeomorphism $\phi \in g$ such that $\phi(C)=C$. Since $\phi\left(\partial D_{n}\right)=\partial D_{n}$, we have $\phi(M)=M$, so that the class $\left[\left.\phi\right|_{M}\right] \in \operatorname{MCG}(M)=\operatorname{MCG}\left(D_{m}\right)$ is well defined.

A similar situation was considered in the proof of Theorem 10.2, where it was shown that under the given assumptions we have $\omega(\beta)=\omega\left(\left[\left.\phi\right|_{M}\right]\right)$. (The arguments used in that proof also imply that the braid $\widehat{\beta}$ is a satellite of the braid $\widehat{\alpha}$ corresponding to the class of the autohomeomorphism $\left.\phi\right|_{M}$.) Since the autohomeomorphism $\left.\phi\right|_{M}$ is of pseudo-Anosov or periodic type, Subsections 11.4 .1 and 11.4.2 imply that $\omega\left(\left[\left.\phi\right|_{M}\right]\right) \in \mathbb{Q}_{[m]}$. Therefore, $\omega(\beta) \in \mathbb{Q}_{[m]} \subset \mathbb{Q}_{[n]}$.

11.5. Remark. A more careful analysis shows that for a reducible braid $\beta \in B_{n}$ (as well as for a pseudo-Anosov one) we have $\omega(\beta) \in \mathbb{Q}_{[n-2]}$.

Indeed, in the notation of Subsection 11.4.3 we have the following: if $m \leq n-2$, then

$$
\omega(\beta)=\omega\left(\left[\left.\phi\right|_{M}\right]\right) \in \mathbb{Q}_{[m]} \subset \mathbb{Q}_{[n-2]} ;
$$

and if $m=n-1$ and $\left[\left.\phi\right|_{M}\right]$ is a class of pseudo-Anosov type, then the arguments used in Subsection 11.4.2 imply that

$$
\omega(\beta)=\omega\left(\left[\left.\phi\right|_{M}\right]\right) \in \mathbb{Q}_{[m-2]} \subset \mathbb{Q}_{[n-3]} .
$$

It remains to consider the case where $m=n-1$ and the class $\left[\left.\phi\right|_{M}\right]$ is of periodic type. In this case, the condition $m=n-1$ implies that the $\phi$-invariant submanifold $C \subset D_{n}$ consists of one component (the interior of which contains precisely two punctures of $D_{n}$ ). This means that $\phi$ preserves the only component of $C$, i.e., $\left[\left.\phi\right|_{M}\right]$ preserves the puncture of $M$ corresponding to the curve $C$. We use the arguments presented in Subsection 11.4.1. Since the autohomeomorphisms in the class $\left[\left.\phi\right|_{M}\right]$ preserve one of the punctures, $\left[\left.\phi\right|_{M}\right]$ falls into case ii). Therefore, the order of the element $\operatorname{mc}\left(\left[\left.\phi\right|_{M}\right]\right) \in \operatorname{mcg}(M)=\operatorname{mcg}\left(D_{m}\right)$ divides $m-1$, i.e.,

$$
\omega(\beta)=\omega\left(\left[\left.\phi\right|_{M}\right]\right) \in \mathbb{Q}_{[m-1]}=\mathbb{Q}_{[n-2]} .
$$

11.6. Assertion. We have $\omega\left(B_{n}\right) \supset \mathbb{Q}_{[n]}$.

Proof. We use the following standard designation:

$$
\delta:=\sigma_{1} \sigma_{2} \cdots \sigma_{n-1} \in B_{n} .
$$

As we know, $\delta^{n}=\Delta^{2}$. For $m \leq n$, we define

$$
\rho_{n, m}:=\delta \sigma_{n-1} \cdots \sigma_{m+1} \sigma_{m} \in B_{n} .
$$

$\left(\right.$ Here, $\rho_{n, n}=\delta$.)

We shall show that for any $k \in \mathbb{Z}$ and any $m \in\{1, \ldots, n\}$ we have

$$
\omega\left(\rho_{n, m}^{k}\right)=\frac{k}{m} .
$$

By property $6.4 .2^{\prime}$, for fixed $m$ (and $n$ ) it suffices to prove the above relation for some nonzero value of $k$. For example, it suffices to prove one of the following two relations:

$$
\omega\left(\rho_{n, m}\right)=\frac{1}{m} \quad \text { and } \quad \omega\left(\rho_{n, m}^{m}\right)=1 .
$$

In the case where $m=n$, the relation $\omega\left(\rho_{n, n}^{n}\right)=1$ follows from the familiar relation $\Delta^{2}=\delta^{n}\left(=\rho_{n, n}^{n}\right)$ and Lemma 5.1.

In the case where $m=n-1$, the obvious relation $\sigma_{i} \delta=\delta \sigma_{i-1}($ for $i \in\{2, \ldots, n-1\})$ 
implies that

$$
\rho_{n, n-1}^{n-1}=\left(\delta \sigma_{n-1}\right)^{n-1}=\delta^{n-1} \sigma_{1} \sigma_{2} \cdots \sigma_{n-1}=\delta^{n}=\Delta^{2},
$$

whence $\omega\left(\rho_{n, n-1}^{n-1}\right)=\omega\left(\Delta^{2}\right)=1$.

In the case where $m \leq n-2$, the closed braid $\widehat{\rho}_{n, m}$ is a satellite of the closed braid $\widehat{\rho}_{m+1, m} \subset B_{m+1}$. (This is easy to check if we observe that the class of autohomeomorphisms of $D_{n}$ corresponding to the braid $\rho_{n, m}$ preserves the class of a simple curve without inflection points and with interior containing the punctures with numbers $m+1, \ldots, n$ (see Subsection 1.3): "gluing together" the strands of the closed braid $\widehat{\rho}_{n, m}$ corresponding to these punctures into one strand, we obtain a closed braid $\widehat{\rho}_{m+1, m}$.) By Theorem 10.2 and the case (considered above) where $m=n-1$ (i.e., $n=m+1$ ), this implies that $\omega\left(\rho_{n, m}\right)=\omega\left(\rho_{m+1, m}\right)=1 / m$.

Thus, we have

$$
\begin{aligned}
\omega\left(B_{n}\right) \supset & \left\{\omega\left(\rho_{n, m}^{k}\right) \mid k \in \mathbb{Z}, m \in\{1, \ldots, n\}\right\} \\
& =\left\{\frac{k}{m} \mid k \in \mathbb{Z}, m \in\{1, \ldots, n\}\right\} \stackrel{\text { def }}{=} \mathbb{Q}_{[n]} .
\end{aligned}
$$

Theorem 11.1 is proved.

\section{§12. Computation of the twist number}

In practice, the twist number of a braid can be computed, for example, with the help of the known algorithms for comparison of braids in Dehornoy's ordering (see, e.g., 5, 12]). Indeed, these algorithms allow us to compute the Dehornoy floor in the braid group. The following proposition shows that this suffices for finding the twist number of any braid.

12.1. Proposition. For each $\beta \in B_{n}$ and $N>n^{2}-n$, we have

$$
\{\omega(\beta)\}=\left[\frac{\left[\beta^{N}\right]_{D}}{N}, \frac{\left[\beta^{N}\right]_{D}+1}{N}\right] \cap \mathbb{Q}_{[n]} .
$$

(Here $[\cdot, \cdot] \subset \mathbb{R}$ is a closed interval of the real line.)

Proof. We define $z:=\left[\beta^{N}\right]_{D}$. By Lemma 7.4, $\omega\left(\beta^{N}\right) \in[z, z+1]$. Since the twist number is a pseudocharacter, we have $\omega(\beta)=\omega\left(\beta^{N}\right) / N$. Thus, $\omega(\beta) \in[z / N,(z+1) / N]$. On the other hand, $\omega(\beta) \in \mathbb{Q}_{[n]}$ by Theorem 11.1. However, the interval $[z / N,(z+1) / N]$ has length $1 / N<1 /\left(n^{2}-n\right)$, so that it contains at most one number in $\mathbb{Q}_{[n]}$, which means that this number is $\omega(\beta)$.

\section{§13. Estimates FOR THE TWIST NUMBER}

Our aim in this section is the proof of the following assertion.

13.1. Proposition. 1) Suppose a braid $\beta \in B_{n}$ is represented by a word $W$ containing precisely $r$ occurrences of the generator $\sigma_{i}$ and $s$ occurrences of the generator $\sigma_{i}^{-1}$ for some $i \in\{1, \ldots, n-1\}$. Then

$$
-s \leq \omega(\beta) \leq r .
$$

2) Suppose, moreover, that $n \geq 4$ and $i \neq 1, n-1$. Then

$$
-\frac{s}{2} \leq \omega(\beta) \leq \frac{r}{2} \text {. }
$$


13.2. Remarks. 1. In the course of the proof of Theorem 6.3, we established (for $n>2$ ) that $\omega\left(\rho^{2} \sigma_{n-1}\right)=1$, where $\rho^{2}$ is a braid written in the generators $\sigma_{1}, \ldots, \sigma_{n-2}$. Thus, the braid $\left(\rho^{2} \sigma_{n-1}\right)^{r}$ with twist number $r$ is represented by a word containing precisely $r$ occurrences of the generator $\sigma_{n-1}$. This means that for $n>2$ the estimate of assertion 1) of Proposition 13.1 is sharp in the case where $i=n-1$, and hence also in the case where $i=1$.

2. In the proof of Assertion 11.6, we established that the twist number of the braid

$$
\rho_{n, 1}=\sigma_{1} \sigma_{2} \cdots \sigma_{n-2} \sigma_{n-1} \sigma_{n-1} \sigma_{n-2} \cdots \sigma_{2} \sigma_{1} \in B_{n}
$$

is equal to 1 . Thus, the braid $\rho_{n, 1}^{r}$ with twist number $r$ is represented by a word containing precisely $2 r$ occurrences of the generator $\sigma_{i}$ for each $i \in\{1, \ldots, n-1\}$. This means that the estimate of assertion 2) of Proposition 13.1 is sharp.

For the proof of assertion 1) of Proposition 13.1, we need the following result.

13.3. Lemma. For any $\beta_{1}, \beta_{2} \in B_{n}$ and any $i \in\{1, \ldots, n-1\}$, we have

$$
\omega\left(\beta_{1} \sigma_{i} \beta_{2}\right) \leq \omega\left(\beta_{1} \beta_{2}\right)+1 .
$$

Proof. This is obvious for $n=2$. For $n>2$, the braid $\sigma_{i} \in B_{n}$ is split, and by Lemma 5.4 we have $\omega\left(\sigma_{i}\right)=0$. Since the twist number is a conjugacy invariant (property 6.4.3), and since its defect is 1 (property 6.4.1), we obtain

$$
\omega\left(\beta_{1} \sigma_{i} \beta_{2}\right)=\omega\left(\beta_{2} \beta_{1} \sigma_{i}\right) \leq \omega\left(\beta_{2} \beta_{1}\right)+\omega\left(\sigma_{i}\right)+1=\omega\left(\beta_{1} \beta_{2}\right)+0+1 .
$$

Proof of assertion 1) of Proposition 13.1. This follows from Corollary 5.5 and Lemma 13.3 .

Proof of assertion 2) of Proposition 13.1. We prove the inequality $\omega(\beta) \leq r / 2$. (The inequality $-s / 2 \leq \omega(\beta)$ is proved in a similar way.)

We need some notation. Let $W$ denote a word representing the given braid $\beta$ and containing precisely $r$ occurrences of the generator $\sigma_{i}$. We denote by $V$ the word in the generators $\sigma_{1}, \ldots, \sigma_{n-1}$ that is obtained from $W$ by deleting all occurrences of the generators $\sigma_{1}^{-1}, \ldots, \sigma_{n-1}^{-1}$. (Like $W$, the word $V$ contains precisely $r$ occurrences of $\sigma_{i}$.) Let $\alpha \in B_{n}$ be the braid represented by $V$. Lemma 5.2 implies that $\omega(\beta) \leq \omega(\alpha)$.

We use induction on the index $n$ of the braid $\beta$. (The base step here is the case where $n=4$.)

Suppose $n=4$ (in this case, we have $i=2$ ). We denote by $U$ the word obtained from $V$ by replacing each occurrence of the letter $\sigma_{2}$ in $V$ by the word $\sigma_{1} \sigma_{2} \sigma_{3} \sigma_{1} \sigma_{2} \sigma_{1}$ (representing the braid $\Delta \in B_{4}$ ). Let $\gamma \in B_{4}$ be the braid represented by $U$. Then $\omega(\alpha) \leq \omega(\gamma)$ by Lemma 5.2. Since in the group $B_{4}$ we have the relations $\sigma_{1} \Delta=\Delta \sigma_{3}$ and $\sigma_{3} \Delta=\Delta \sigma_{1}$, and the generators $\sigma_{1}$ and $\sigma_{3}$ commute, it follows that $\gamma=\Delta^{r} \sigma_{1}^{x} \sigma_{3}^{y}$ for some $x, y \in \mathbb{Z}$. Obviously, the braid $\Delta^{r} \sigma_{1}^{x} \sigma_{3}^{y} \in B_{4}$ is a satellite of the braid $\sigma_{1}^{r} \in B_{2}$, whence $\omega(\gamma)=\omega\left(\sigma_{1}^{r}\right)=r / 2$ by Theorem 10.2. Thus, for $n=4$ we have

$$
\omega(\beta) \leq \omega(\alpha) \leq \omega(\gamma)=\omega\left(\sigma_{1}^{r}\right)=\frac{r}{2} .
$$

Suppose $n \geq 5$. Without loss of generality, we can assume that $i \leq n-3$. (Indeed, in the case where $i=n-3$ we consider the braid $\Delta \alpha \Delta^{-1}$.) We denote by $X$ the braid word obtained by substituting the word $\sigma_{n-2} \sigma_{n-1} \sigma_{n-1} \sigma_{n-2}$ for each letter $\sigma_{n-1}$ or $\sigma_{n-2}$ in the braid word $V$. Let $\alpha_{1} \in B_{n}$ be the braid represented by $X$. Then Lemma 5.2 implies that $\omega(\alpha) \leq \omega\left(\alpha_{1}\right)$. We denote by $Y$ the braid word obtained from $X$ by substituting the word $\sigma_{n-2} \sigma_{n-2}$ for each subword $\sigma_{n-2} \sigma_{n-1} \sigma_{n-1} \sigma_{n-2}$ in $X$. Let $\alpha_{2} \in B_{n-1}$ be the $(n-1)$-strand braid represented by $Y$. We observe that, by construction, $Y$ is a word in the generators $\sigma_{1}, \ldots, \sigma_{n-2}$, and it contains exactly $r$ entries of the letter $\sigma_{i}$. Hence, by the induction hypothesis, we have $\omega\left(\alpha_{2}\right) \leq r / 2$. At the same time, it is easily seen that 
the braid $\alpha_{1}$ is a satellite of the braid $\alpha_{2}$. Then, by Theorem 10.2 , we have $\omega\left(\alpha_{1}\right)=\omega\left(\alpha_{2}\right)$, so that

$$
\omega(\beta) \leq \omega(\alpha) \leq \omega\left(\alpha_{1}\right)=\omega\left(\alpha_{2}\right) \leq \frac{r}{2} .
$$

\section{§14. Transformations of Closed Braids and Menasco's conjectures}

A transformation of a closed braid $\widehat{\alpha}$ is a transition from $\widehat{\alpha}$ to a braid $\widehat{\beta}$ that represents the same link as $\widehat{\alpha}$. Important results of the theory of braids and links are related to the transformations of stabilization and destabilization introduced by Markov, as well as to "exchange move" and "flype" defined by Birman and Menasco (see the definitions below). While stabilization can always be performed, the other transformations mentioned above are not applicable to each braid. No algorithm determining whether or not one of these transformations is applicable to a given closed braid has been found up to now.

Using the Nielsen-Thurston classification of automorphisms of surfaces, Menasco [10] defined certain characteristics of periodic and pseudo-Anosov braids, and in terms of these characteristics he stated four conjectures concerning the applicability of destabilization, exchange move, and flype to a closed braid. It turns out that for braids of both types indicated above, Menasco's characteristics coincide with the twist number of a braid (see the definitions in 10 and $\S 11$ above).

In Theorem 14.2, in terms of the twist number we establish restrictions on the possibility of applying destabilization, exchange move, and flype to closed braids. Theorem 14.2 implies the validity of all four conjectures, and some of them in a stronger form (with the exception of the conjecture on periodic braids in the part concerning flype: as shown in [11, this part is not true).

\subsection{Definitions.}

14.1.1. Destabilization. We say that a closed braid $\widehat{\alpha} \subset B_{n-1}$ of index $n-1$ is obtained from a closed braid $\widehat{\beta} \subset B_{n}$ of index $n$ by destabilization if there is a braid $\gamma \in \widehat{\alpha}$ such that $\gamma \sigma_{n-1} \in \widehat{\beta}$ or $\gamma \sigma_{n-1}^{-1} \in \widehat{\beta}$ :

$$
\widehat{\beta} \ni \gamma \sigma_{n-1}^{ \pm 1} \longmapsto \gamma \in \widehat{\alpha} .
$$

Thus, $\widehat{\beta}$ admits destabilization if $\widehat{\beta}$ contains a braid that can be written with only one (in total) occurrence of the generators $\sigma_{n-1}$ and $\sigma_{n-1}^{-1}$.

14.1.2. Exchange move. Suppose $\widehat{\alpha}, \widehat{\beta} \subset B_{n}$ are two closed braids of index $n$. We say that $\widehat{\alpha}$ is obtained from $\widehat{\beta}$ by an exchange move if there are $\gamma_{1}, \gamma_{2} \in B_{n-1} \subset B_{n}$ such that $\gamma_{1} \sigma_{n-1} \gamma_{2} \sigma_{n-1}^{-1} \in \widehat{\beta}$ and $\gamma_{1} \sigma_{n-1}^{-1} \gamma_{2} \sigma_{n-1} \in \widehat{\alpha}$ :

$$
\widehat{\beta} \ni \gamma_{1} \sigma_{n-1} \gamma_{2} \sigma_{n-1}^{-1} \longmapsto \gamma_{1} \sigma_{n-1}^{-1} \gamma_{2} \sigma_{n-1} \in \widehat{\alpha} .
$$

Thus, a closed braid $\widehat{\beta}$ admits an exchange move if $\widehat{\beta}$ contains a braid word of the form $U \sigma_{n-1} V \sigma_{n-1}^{-1}$, where $U$ and $V$ are words in the generators $\sigma_{1}^{ \pm 1}, \ldots, \sigma_{n-2}^{ \pm 1}$.

14.1.3. Flype. Since the algebraic definition of flype is rather awkward, we define only the admissibility of this transformation. (See [13] for the consistency of this definition.) We say that a closed braid $\widehat{\beta}$ of index $n$ admits a flype if $\widehat{\beta}$ contains a braid word of the form $U \sigma_{n-1} V \sigma_{n-1}^{ \pm 1} W \sigma_{n-1}^{-1}$, where $U$ and $V$ are words in the generators $\sigma_{1}^{ \pm 1}, \ldots, \sigma_{n-2}^{ \pm 1}$. The above definitions and assertion 1) of Proposition 13.1 immediately imply the following result. 
14.2. Theorem. Suppose $\beta \in B_{n}$.

1) If $|\omega(\beta)|>1$, then the closed braid $\widehat{\beta}$ admits neither destabilization, nor exchange move.

2) If $|\omega(\beta)|>2$, then the closed braid $\widehat{\beta}$ does not admit a flype.

14.3. Remarks. 1. The restrictions on the admissibility of transformations that are provided by Theorem 14.2 can be somewhat refined in terms of orderings on the braid group (see [13, Theorem 5.1]).

2. In terms of the twist number, we can also obtain restrictions on other transformations of closed braids. (We do not present these restrictions here; they automatically follow from the proved properties of the twist number and the results of [13.)

\section{$\S 15$. THE TWIST NUMBER AND LINKS}

Closed braids representing the same link may have different twist numbers, so that the twist number is not an invariant of a link. Nevertheless, the twist number of a braid provides some information on the corresponding link. For example, in Theorem 15.3 below, in terms of the twist number we establish sufficient conditions of primality of the link represented by a braid.

15.1. We recall some notions of link theory. A link $L \subset S^{3}$ is trivial if there is a sphere $S^{2} \subset S^{3}$ such that $L \subset S^{2}$. A link $L \subset S^{3}$ is split if there is a sphere $S^{2} \subset S^{3} \backslash L$ that does not bound a ball (in $S^{3} \backslash L$ ). A link $L \subset S^{3}$ is composite if there is a sphere $S^{2} \subset S^{3}$ that intersects the link $L$ at two points and splits it into two links ("tangles") such that none of them is an unknotted arc. A link is prime if it is not either composite, or split, or trivial.

We denote by $\mathcal{L}(\beta)$ an oriented link represented by a braid $\widehat{\beta}$.

15.2. Proposition [13. Suppose $\beta \in B_{n}$, where $n>2$, and suppose that the link $\mathcal{L}(\beta)$ is not prime. Then the closed braid $\widehat{\beta}$ admits an exchange move.

Combining this with Theorem 14.2, we obtain the following (the case where $n=2$ is trivial).

15.3. Theorem. Suppose $\beta \in B_{n}$. If $|\omega(\beta)|>1$, then $\mathcal{L}(\beta)$ is a prime (i.e., noncomposite, nonsplit, and nontrivial) link.

15.4. Remark. Sufficient conditions of primality for a link that are given by Theorem 15.3 can be refined somewhat in terms of orderings on the braid group (see [13, Theorem $6.2])$.

\section{REFERENCES}

[1] J. S. Birman, Braids, links, and mapping class groups, Ann. of Math. Stud., No. 82, Princeton Univ. Press, Princeton, NJ, 1974. MR0375281 (51:11477)

[2] L. E. J. Brouwer, Über die periodischen Transformationen der Kugel, Math. Ann. 80 (1919), 39-41.

[3] A. Casson and S. Bleiler, Automorphisms of surfaces after Nielsen and Thurston, London Math. Soc. Stud. Texts, vol. 9, Cambridge Univ. Press, Cambridge, 1988. MR0964685 (89k:57025)

[4] A. Constantin and B. Kolev, The theorem of Kerékjártó on periodic homeomorphisms of the disc and the sphere, Enseign. Math. (2) 40 (1994), 193-204. MR1309126 (95j:55005)

[5] P. Dehornoy, I. Dynnikov, D. Rolfsen, and B. Wiest, Why are braids orderable?, Panor. Synthèses, vol. 14, Soc. Math. France, Paris, 2002. MR1988550(2004e:20062)

[6] V. A. Faĭziev, The stability of the equation $f(x y)-f(x)-f(y)=0$ on groups, Acta Math. Univ. Comenian. (N.S.) 69 (2000), no. 1, 127-135. MR1796793 (2003a:39026)

[7] É Ghys, Groups acting on the circle, Enseign. Math. (2) 47 (2001), 329-407. MR.1876932 (2003a:37032) 
[8] R. Grigorchuk, Some results on bounded cohomology, Combinatorial and Geometric Group Theory (Edinburgh, 1993), London Math. Soc. Lecture Note Ser., vol. 204, Cambridge Univ. Press, Cambridge, 1995, pp. 111-163. MR.1320279 (96j:20073)

[9] B. von Kerékjártó, Über die periodischen Transformationen der Kreisscheibe und der Kugelfläche, Math. Ann. 80 (1919), 36-38.

[10] R. Kirby (ed.), Problems in low-dimensional topology, Geometric Topology (Athens, GA, 1993), AMS/IP Stud. Adv. Math., vol. 2.2, Amer. Math. Soc., Providence, RI, 1997, pp. 35-473. MR 1470751

[11] A. V. Malyutin, Orderings on braid groups, operations over closed braids, and confirmation of Menasco's conjecture, Zap. Nauchn. Sem. S.-Peterburg. Otdel. Mat. Inst. Steklov. (POMI) 267 (2000), 163-169; English transl., J. Math. Sci. 113 (2003), no. 6, 822-826. MR1809825 (2002a:57008)

[12] , Fast algorithms for the recognition and comparison of braids, Zap. Nauchn. Sem. S.Peterburg. Otdel. Mat. Inst. Steklov. (POMI) 279 (2001), 197-217; English transl., J. Math. Sci. 119 (2004), no. 1, 101-111. MR 1846081 (2002g:20061)

[13] A. V. Malyutin and N. Yu. Netsvetaev, Dehornoy's ordering on the braid group and braid moves, Algebra i Analiz 15 (2003), no. 3, 170-187; English transl., St. Petersburg Math. J. 15 (2004), no. 3, 437-448. MR2052167 (2005b:20068)

[14] H. Short and B. Wiest, Orderings of mapping class groups after Thurston, Enseign. Math. (2) 46 (2000), 279-312. MR 1805402 (2003b:57003)

[15] W. P. Thurston, On the geometry and dynamics of diffeomorphisms of surfaces, Bull. Amer. Math. Soc. (N.S.) 19 (1988), 417-431. MR0956596 (89k:57023)

[16] A. Fathi, F. Laudenbach, and V. Poenaru (eds.), Travaux de Thurston sur les surfaces, Séminaire Orsay, Astérisque, vol. 66-67, Soc. Math. France, Paris, 1979. MR0568308 (82m:57003)

St. Petersburg Branch, Steklov Mathematical Institute, Russian Academy of Sciences, Fontanka 27, St. Petersburg 191023, Russia

E-mail address: malyutin@pdmi.ras.ru

Received 31/JAN/2003

Translated by N. YU. NETSVETAEV 